\title{
An Extension to a Theorem of Jörgens, Calabi, and Pogorelov
}

\author{
L. CAFFARELLI \\ University of Texas \\ AND \\ YANYAN LI \\ Rutgers-The State University
}

\section{Introduction}

A classical theorem of Jörgens ( $n=2$ [17]), Calabi $(n \leq 5$ [7]), and Pogorelov $(n \geq 2[18])$ states that any classical convex solution of

$$
\operatorname{det}\left(D^{2} u\right)=1 \quad \text { in } \mathbb{R}^{n}
$$

must be a quadratic polynomial. For $n=2$, a classical solution is either convex or concave; the result holds without the convexity hypothesis.

A simpler and more analytical proof, along the lines of affine geometry, of the theorem was later given by Cheng and Yau [9]. The first author extended the result for classical solutions to viscosity solutions [4]. It was proven by Trudinger and Wang in [19] that the only open convex subset $\Omega$ of $\mathbb{R}^{n}$ which admits a convex $C^{2}$ solution of $\operatorname{det}\left(D^{2} u\right)=1$ in $\Omega$ with $\lim _{x \rightarrow \partial \Omega} u(x)=\infty$ is $\Omega=\mathbb{R}^{n}$. In this paper we give the following extension to the theorem of Jörgens, Calabi, and Pogorelov: Let $u$ be a convex viscosity solution of $\operatorname{det}\left(D^{2} u\right)=1$ outside a bounded subset of $\mathbb{R}^{n}, n \geq 3$; then there exist an $n \times n$ real symmetric positive definite matrix $A$, a vector $b \in \mathbb{R}^{n}$, and a constant $c \in \mathbb{R}$ such that

$$
\limsup _{|x| \rightarrow \infty}|x|^{n-2}\left(u-\left[\frac{1}{2} x^{\prime} A x+b \cdot x+c\right]\right)<\infty .
$$

Our approach, different from previous ones, is based on the theory of the first author on Monge-Ampère equations [2, 3]. Our above-mentioned results also enable us to establish an existence result for the Dirichlet problem on exterior domains with prescribed asymptotic behavior at infinity. In $\mathbb{R}^{2}$, similar problems are studied by L. Ferrer, A. Martínez, and F. Milán in [13, 14] using complex variable methods. See also Delanoë [12].

For the reader's convenience, we recall the definition of viscosity solutions (see [5] and the references therein for an extensive study of viscosity solutions to fully nonlinear elliptic equations of second order). Let $\Omega$ be an open subset of $\mathbb{R}^{n}$, 
$g \in C^{0}(\Omega)$ a positive function, and $u \in C^{0}(\Omega)$ a locally convex function. We say that $u$ is a viscosity subsolution of

$$
\operatorname{det}\left(D^{2} u\right)=g \quad \text { in } \Omega
$$

or a viscosity solution of

$$
\operatorname{det}\left(D^{2} u\right) \geq g \quad \text { in } \Omega
$$

if for every $\bar{x} \in \Omega$ and every convex $\varphi \in C^{2}(\Omega)$ satisfying

$$
\varphi \geq u \text { on } \Omega \text { and } \varphi(\bar{x})=u(\bar{x})
$$

we have

$$
\operatorname{det}\left(D^{2} \varphi(\bar{x})\right) \geq g(\bar{x}) .
$$

Similarly, $u$ is a viscosity supersolution of (1.2) if for every $\bar{x} \in \Omega$ and every convex $\varphi \in C^{2}(\Omega)$ satisfying

$$
\varphi \leq u \text { on } \Omega \quad \text { and } \quad \varphi(\bar{x})=u(\bar{x})
$$

we have

$$
\operatorname{det}\left(D^{2} \varphi(\bar{x})\right) \leq g(\bar{x}) .
$$

$u$ is a viscosity solution of (1.2) if $u$ is both a viscosity subsolution and a viscosity supersolution of (1.2).

In this paper we study convex viscosity solutions to

$$
\operatorname{det}\left(D^{2} u\right)=f \quad \text { on } \mathbb{R}^{n},
$$

where $f \in C^{0}\left(\mathbb{R}^{n}\right)$ satisfies

$$
0<\inf _{\mathbb{R}^{n}} f \leq \sup _{\mathbb{R}^{n}} f<\infty
$$

and

$$
\text { support }(f-1) \text { is bounded. }
$$

First is the extension of the classical theorem of Jörgens, Calabi, and Pogorelov for classical solutions to viscosity solutions, due to the first author.

THEOREM 1.1 [4] For $n \geq 2$, any convex viscosity solution of (1.1) must be a quadratic polynomial.

\section{Let}

$$
\mathcal{A}=\{A: A \text { is real } n \times n \text { symmetric positive definite matrix with } \operatorname{det}(A)=1\} \text {. }
$$

The following theorem gives asymptotic behavior of solutions of (1.3) under the hypotheses (1.4) and (1.5).

THEOREM 1.2 Let $f \in C^{0}\left(\mathbb{R}^{n}\right)$ satisfy (1.4) and (1.5). Assume that $u$ is a convex viscosity solution of (1.3). Then 
(i) For $n \geq 3$, there exist some $c \in \mathbb{R}, b \in \mathbb{R}^{n}$, and $A \in \mathcal{A}$, such that $E(x):=$ $u(x)-\left(\frac{1}{2} x^{\prime} A \bar{x}+b \cdot x+c\right)$ satisfies

$$
\limsup _{|x| \rightarrow \infty}|x|^{n-2}|E(x)|<\infty .
$$

Moreover, $u$ is $C^{\infty}$ in the complement of the support of $(f-1)$ and

$$
\limsup _{|x| \rightarrow \infty}|x|^{n-2+k}\left|D^{k} E(x)\right|<\infty \quad \forall k \geq 1 .
$$

(ii) For $n=2$, there exist some $c \in \mathbb{R}, b \in \mathbb{R}^{n}$, and $A \in \mathcal{A}$ such that

$$
E(x):=u(x)-\left(\frac{1}{2} x^{\prime} A x+b \cdot x+d \log \sqrt{x^{\prime} A x}+c\right)
$$

satisfies

$$
\limsup _{|x| \rightarrow \infty}|x||E(x)|<\infty
$$

where

$$
d=\frac{1}{2 \pi} \int_{\mathbb{R}^{2}}(f-1)
$$

Moreover, $u$ is $C^{\infty}$ in the complement of the support of $(f-1)$ and

$$
\limsup _{|x| \rightarrow \infty}|x|^{k+1}\left|D^{k} E(x)\right|<\infty \quad \forall k \geq 1 \text {. }
$$

COROLlaRY 1.3 Let $O$ be a bounded open convex subset of $\mathbb{R}^{n}$, and let $u \in$ $C^{0}\left(\mathbb{R}^{n} \backslash \bar{O}\right)$ be a locally convex viscosity solution of

$$
\operatorname{det}\left(D^{2} u(x)\right)=1, \quad x \in \mathbb{R}^{n} \backslash \bar{O} .
$$

Then $u \in C^{\infty}\left(\mathbb{R}^{n} \backslash \bar{O}\right)$, and we have the following:

(i) For $n \geq 3$, there exist some $c \in \mathbb{R}, b \in \mathbb{R}^{n}$, and $A \in \mathcal{A}$ such that (1.6) and (1.7) hold.

(ii) For $n=2$, there exist some $c, d \in \mathbb{R}, b \in \mathbb{R}^{2}$, and $A \in \mathcal{A}$ such that (1.8) and (1.10) hold. Moreover, if $O=\varnothing$, then $d=0$.

Remark 1.4. For $n=2$, Corollary 1.3 is known (see [14]).

The theorem of Jörgens, Calabi, and Pogorelov is an easy consequence of Corollary 1.3. Indeed, let $u \in C^{2}$ be a convex solution of (1.1). Then, by Corollary 1.3 , for some $c, b$, and $A \in \mathcal{A}$,

$$
E(x):=u(x)-\left(\frac{1}{2} x^{\prime} A x+b \cdot x+c\right) \rightarrow 0 \quad \text { as }|x| \rightarrow \infty .
$$

Since

$$
\operatorname{det}\left(A+D^{2} E\right)-\operatorname{det}(A)=\operatorname{det}\left(D^{2} u\right)-1=0
$$


and $\left(A+D^{2} E\right)=\left(D^{2} u\right)$ is positive definite, it follows from the mean value theorem that for some positive definite matrix function $\left(a_{i j}(x)\right)$,

$$
a_{i j} D_{i j} E=0 \text { in } \mathbb{R}^{n} \text {. }
$$

By the maximum principle, $E(x) \equiv 0$, i.e., $u(x) \equiv \frac{1}{2} x^{\prime} A x+b \cdot x+c$.

Corollary 1.3 enables us to establish the following existence theorem for the Dirichlet problem on exterior domains with prescribed asymptotic behavior at infinity.

THEOREM 1.5 Let $D$ be a smooth, bounded, strictly convex open subset of $\mathbb{R}^{n}$, $n \geq 3$, and let $\varphi \in C^{2}(\partial D)$. Then for any given $b \in \mathbb{R}^{n}$ and any given $A \in \mathcal{A}$, there exists some constant $c_{*}$, depending only on $n, D, \varphi, b$, and $A$, such that for every $c>c_{*}$ there exists a unique function $u \in C^{\infty}\left(\mathbb{R}^{n} \backslash \bar{D}\right) \cap C^{0}\left(\overline{\mathbb{R}^{n} \backslash D}\right)$ that satisfies

$$
\begin{cases}\operatorname{det}\left(D^{2} u\right)=1,\left(D^{2} u\right)>0, & \text { in } \mathbb{R}^{n} \backslash \bar{D} \\ u=\varphi & \text { on } \partial D\end{cases}
$$

and

$$
\limsup _{|x| \rightarrow \infty}\left(|x|^{n-2}\left|u(x)-\left[\frac{1}{2} x^{\prime} A x+b \cdot x+c\right]\right|\right)<\infty \text {. }
$$

Remark 1.6. The Dirichlet problem on exterior domains of $\mathbb{R}^{2}$ was studied by Ferrer, Martínez, and Milán in [13, 14] using complex variable methods.

Our next theorem gives the existence of solutions of (1.3) with given asymptotic behavior at infinity.

THEOREM 1.7 For $n \geq 3$, let $f \in C^{0}\left(\mathbb{R}^{n}\right)$ satisfy (1.4) and (1.5). Then for any $c \in \mathbb{R}, b \in \mathbb{R}^{n}$, and $A \in \mathcal{A}$, there exists a unique convex viscosity solution $u$ of (1.3) satisfying

$$
\lim _{|x| \rightarrow \infty} E(x)=0,
$$

where $E(x)=u(x)-\left(\frac{1}{2} x^{\prime} A x+b \cdot x+c\right)$. Moreover, $u$ is $C^{\infty}$ in the complement of the support of $(f-1)$, and $E$ satisfies

$$
\left(1+|x|^{n-2}\right)|E(x)| \leq C, \quad x \in \mathbb{R}^{n},
$$

In addition, for any $k \geq 1$,

$$
|x|^{n-2+k}\left|D^{k} E(x)\right| \leq C, \quad|x| \geq r,
$$

where $C$ and $r$ are some positive constants depending only on $n, f$, and $k$.

Remark 1.8. Let $u$ be the convex viscosity solution in Theorem 1.7. Then for $M>C,\{x: u(x)<M\}$ contains a ball of radius $\sqrt{2(M-C)}$ and is contained in a ball of radius $\sqrt{2(M+C)}$. By the works of the first author [2,3], $u$ is strictly convex. Moreover, if $f \in C^{k, \alpha}(O)$ for some $k \geq 0,0<\alpha<1$, and some open subset of $\mathbb{R}^{n}$, then $u \in C^{k+2, \alpha}(O)$. 
Remark 1.9. For smooth $f$, Theorem 1.7 is a special case of results that Delanoë [12] obtained by different methods. For any general measurable function $f$ satisfying (1.4), existence of infinitely many entire viscosity solutions was established by Chou and Wang in [10].

\section{Proof of Theorem 1.1}

In this section we give a proof of Theorem 1.1, an extension, due to the first author ([4]), of the theorem of Jörgens, Calabi, and Pogorelov for classical solutions to viscosity solutions. We need some well-known comparison principles. For the reader's convenience, we include the simple proofs.

Proposition 2.1 Let $\Omega$ be a bounded open subset of $\mathbb{R}^{n}, n \geq 2$, and let $g \in$ $C^{0}(\Omega)$ be a positive function. Assume that $w \in C^{0}(\bar{\Omega})$ is a locally convex viscosity subsolution (supersolution) of

$$
\operatorname{det}\left(D^{2} w\right)=g \quad \text { in } \Omega,
$$

and $v \in C^{0}(\bar{\Omega}) \cap C^{2}(\Omega)$ is a locally convex supersolution (subsolution) of

$$
\operatorname{det}\left(D^{2} v\right)=g \text { in } \Omega \text {. }
$$

Assume also that

$$
w \leq v(w \geq v) \quad \text { on } \partial \Omega
$$

Then

$$
w \leq v(w \geq v) \quad \text { on } \bar{\Omega} .
$$

PROOF: Without loss of generality, $\bar{\Omega} \subset B_{R}$ for some $R>0$. For $0<\epsilon<1$ and $\lambda \geq 0$, let

$$
v_{\epsilon}(x)=v(x)-\epsilon\left(|x|^{2}-R^{2}\right) \quad \text { and } \quad v_{\epsilon, \lambda}(x)=v_{\epsilon}(x)+\lambda .
$$

For $\lambda$ sufficiently large,

$$
v_{\epsilon, \lambda} \geq w \quad \text { on } \bar{\Omega} .
$$

Let $\bar{\lambda}(\epsilon)$ be the smallest $\lambda \geq 0$ such that the above holds. We will show that

$$
\bar{\lambda}:=\limsup _{\epsilon \rightarrow 0} \bar{\lambda}(\epsilon)=0 \text {. }
$$

Indeed, if $\bar{\lambda}>0$, then there exist $\epsilon_{j} \rightarrow 0^{+}, \bar{\lambda}_{j}:=\bar{\lambda}_{j}\left(\epsilon_{j}\right) \rightarrow \bar{\lambda}$, and $\bar{x}_{j} \in \bar{\Omega}_{\bar{x}} \rightarrow \bar{x}$, such that

$$
v_{\epsilon_{j}, \bar{\lambda}_{j}}\left(\bar{x}_{j}\right)=w\left(\bar{x}_{j}\right)
$$

while

$$
v_{\epsilon_{j}, \bar{\lambda}_{j}} \geq w \text { on } \bar{\Omega}
$$


Since $v_{\epsilon, \bar{\lambda}}>w$ on $\partial \Omega$, we have $\bar{x} \in \Omega$. Since $\operatorname{det}\left(D^{2} v\right)>0$ and $v$ is convex, we have $\left(D^{2} v(\bar{x})\right)>0$, so, for large $j, v_{\epsilon_{j}, \bar{\lambda}_{j}}$ is strictly convex near $\bar{x}_{j}$. By the definition of viscosity solution and taking $v_{\epsilon_{j}, \bar{\lambda}_{j}}$ as a test function, we have

$$
\operatorname{det}\left(D^{2} v_{\epsilon_{j}, \bar{\lambda}_{j}}\left(\bar{x}_{j}\right)\right) \leq g\left(\bar{x}_{j}\right) \text {. }
$$

On the other hand, from the explicit expression of $v_{\epsilon, \bar{\lambda}}$, we have

$$
\operatorname{det}\left(D^{2} v_{\epsilon_{j}, \bar{\lambda}_{j}}\left(\bar{x}_{j}\right)\right)=\operatorname{det}\left(D^{2} v\left(\bar{x}_{j}\right)-2 \epsilon_{j} I\right)<\operatorname{det}\left(D^{2} v\left(\bar{x}_{j}\right)\right) \leq g\left(\bar{x}_{j}\right),
$$

a contradiction. So (2.1) holds, and therefore

$$
w(x) \leq \lim _{\epsilon \rightarrow 0} v_{\epsilon, \bar{\lambda}(\epsilon)}(x)=v(x), \quad x \in \bar{\Omega} .
$$

The statement concerning viscosity supersolution $w$ can be proven in a similar way.

There are two immediate consequences of Proposition 2.1:

COROLLARY 2.2 Let $w \in C^{0}\left(\bar{B}_{R}\right)$ be a locally convex viscosity subsolution of

$$
\operatorname{det}\left(D^{2} w\right)=1 \quad \text { on } B_{R} \text {. }
$$

Then

$$
w(x) \leq \frac{1}{2}\left(|x|^{2}-R^{2}\right)+\max _{\partial B_{R}} w \quad \forall x \in B_{R} .
$$

COROLlaRY 2.3 Let $w \in C^{0}\left(\bar{B}_{R}\right)$ be a locally convex viscosity supersolution of

$$
\operatorname{det}\left(D^{2} w\right)=1 \quad \text { on } B_{R} \text {. }
$$

Then

$$
w(x) \geq \frac{1}{2}\left(|x|^{2}-R^{2}\right)+\min _{\partial B_{R}} w \quad \forall x \in B_{R} .
$$

Proof of COROllary 2.2: The function

$$
v(x):=\frac{1}{2}\left(|x|^{2}-R^{2}\right)+\max _{\partial B_{R}} w
$$

is a $C^{2}$ convex function in $\bar{B}_{R}$ satisfying

$$
\operatorname{det}\left(D^{2} v\right)=1 \text { on } B_{R}, \quad w \leq v \text { on } \partial B_{R} .
$$

Estimate (2.2) follows from Proposition 2.1.

Proof OF COROLlary 2.3: Let

$$
v(x):=\frac{1}{2}\left(|x|^{2}-R^{2}\right)+\min _{\partial B_{R}} w
$$

and apply Proposition 2.1.

The following result is well-known; see, for example, [1] and [8]. 
PROpOSITION 2.4 Let $\Omega$ be a bounded open convex subset of $\mathbb{R}^{n}, n \geq 2$, and let $f \in C^{0}(\bar{\Omega}) \cap C^{\infty}(\Omega)$ be a positive function. Then there exists a convex solution $w \in C^{0}(\bar{\Omega}) \cap C^{\infty}(\Omega)$ of

$$
\begin{cases}\operatorname{det}\left(D^{2} w\right)=f & \text { in } \Omega \\ w=0 & \text { on } \partial \Omega\end{cases}
$$

Remark 2.5. The locally convex viscosity solution to the above Dirichlet problem is unique. This follows from Proposition 2.1.

Remark 2.6. If $\Omega$ is a $C^{\infty}$ strictly convex domain and $f \in C^{\infty}(\bar{\Omega})$ is positive, then, by [6], $w \in C^{\infty}(\bar{\Omega})$.

To prove Theorem 1.1, we will first show that a convex viscosity solution of (1.1) is a classical solution.

THEOREM 2.7 Let $u$ be a convex viscosity solution of (1.1). Then $u \in C^{\infty}\left(\mathbb{R}^{n}\right)$.

For $M>0$, let $\Omega_{M}=\left\{x \in \mathbb{R}^{n}: u(x)<M\right\}$.

PROPOSITION 2.8 Let $u$ be a nonnegative convex viscosity subsolution of (1.1). Then there exists some constant $C$, depending only on $n$, such that

$$
\Omega_{M} \subset B_{C M^{n / 2}} \quad \forall M \geq \max _{\partial B_{1}} u \text {. }
$$

Proposition 2.8 will be deduced from Corollary 2.2 and the lemma below. Let $e_{1}=(0, \ldots, 0,1)$ and, for $\delta>0, B_{\delta}^{\prime}=\left\{\left(0, x_{2}, \ldots, x_{n}\right):\left|\left(0, x_{2}, \ldots, x_{n}\right)\right|<\delta\right\}$.

LEMMA 2.9 For $\delta, r, \lambda>0$, let $K$ be the convex hull of $\overline{B_{\delta}^{\prime}} \cup\left\{r e_{1}\right\}$, and let $u \in$ $C^{0}(K)$ be a nonnegative convex viscosity solution of

$$
\operatorname{det}\left(D^{2} u\right) \geq \lambda \quad \text { in the interior of } K .
$$

Assume, for some positive constant $\beta$, that

$$
u \leq \beta \quad \text { on } \bar{B}_{\delta}^{\prime} .
$$

Then, for some constant $C=C(n) \geq 1$,

$$
\max \left\{\beta, u\left(r e_{1}\right)\right\} \geq \frac{\lambda^{1 / n} \delta^{\frac{2(n-1)}{n}} r^{2 / n}}{C} .
$$

PROOF: By the convexity of $u$,

$$
\max _{K} u \leq \alpha:=\max \left\{\beta, u\left(r e_{1}\right)\right\} .
$$

It is clear that we can put an ellipsoid $E$ in $K$ with

$$
|E| \geq \frac{\delta^{n-1} r}{C}
$$


for some $C=C(n) \geq 1$. For some $a \in \mathcal{A}, b \in \mathbb{R}^{n}$, and

$$
A(x)=a x+b,
$$

we have, for some $R>0$,

$$
A(E)=B_{R} .
$$

Consider

Then

$$
w(x)=\frac{1}{\lambda^{1 / n}} u\left(A^{-1}(x)\right), \quad x \in B_{R} .
$$

in the viscosity sense, and

$$
\operatorname{det}\left(D^{2} w\right) \geq 1 \quad \text { in } B_{R}
$$

$$
w \leq \frac{\alpha}{\lambda^{1 / n}} \quad \text { on } \partial B_{R} .
$$

It follows from Corollary 2.2 and the fact that $u \geq 0$ that

$$
0 \leq w(0) \leq-\frac{1}{2} R^{2}+\max _{\partial B_{R}} w \leq-\frac{1}{2} R^{2}+\frac{\alpha}{\lambda^{1 / n}} .
$$

Thus

$$
\delta^{n-1} r \leq C(n)|E| \leq C(n) R^{n} \leq \frac{C(n) \alpha^{n / 2}}{\lambda^{1 / 2}} .
$$

Lemma 2.9 follows from the above.

Proof of Proposition 2.8: For $M \geq \max _{\partial B_{1}} u$, without loss of generality, we may assume that

$$
u\left(r e_{1}\right)=M .
$$

We need to show that $r \leq C(n) M^{n / 2}$. By Corollary 2.2, $\max _{\partial B_{1}} u \geq \frac{1}{2}$. So we only need to consider $r \geq 2$. Let $\widetilde{K}$ denote the convex hull of $\bar{B}_{1}$ and $r e$. By the convexity of $u$ and the fact that the values of $u$ on $\partial B_{1}$ and at $r e$ are bounded by $M$, we have

$$
\max _{\widetilde{K}} u \leq M .
$$

Proposition 2.8 follows from Lemma 2.9 (with $K$ being the convex hull of $\widetilde{K} \cap$ $\left\{x_{1}=1\right\}$ and $\left.r e_{1}\right)$.

Let $u$ be a nonnegative convex viscosity subsolution of (1.1). By Proposition 2.8, $\Omega_{M}$ is bounded and convex for every $M>\inf _{\mathbb{R}^{n}} u$. By a normalization lemma of John-Cordoba and Gallegos (see [11]), there exists some affine transformation

$$
A_{M}(x)=a_{M} x+b_{M},
$$

where $a_{M}$ is an $n \times n$ matrix satisfying

$$
\operatorname{det}\left(a_{M}\right)=1
$$

and $b_{M} \in \mathbb{R}^{n}$ such that

$$
B_{R} \subset A_{M}\left(\Omega_{M}\right) \subset B_{n R} \text { for some } R>0 .
$$


PROPOSITION 2.10 Let $0<\lambda<\infty$, and let $u$ be a convex viscosity solution of

$$
\operatorname{det}\left(D^{2} u\right) \geq \lambda \quad \text { on } \mathbb{R}^{n} .
$$

Assume that $u$ is normalized to further satisfy

$$
u(0)=0, \quad u \geq 0 \text { on } \mathbb{R}^{n} .
$$

Then there exists some constant $C \geq 1$ depending only only $n$ such that

$$
\left|\Omega_{M}\right| \leq C \lambda^{-1 / 2} M^{n / 2} \quad \forall M>0 .
$$

PROOF: Let

$$
A_{M}(x)=a_{M} x+b_{M}
$$

be an affine transformation satisfying (2.3) and (2.4). Consider

$$
w(x)=\lambda^{-1 / n} u\left(A_{M}^{-1}(x)\right) .
$$

Then, by (2.5),

$$
\operatorname{det}\left(D^{2} w\right) \geq 1 \text { on } \mathbb{R}^{n} \quad \text { and } \quad w \leq \lambda^{-1 / n} M \text { on } \partial B_{R} .
$$

Applying Corollary 2.2, we have

$$
0 \leq w(0) \leq-\frac{1}{2} R^{2}+\max _{\partial B_{R}} w \leq-\frac{1}{2} R^{2}+\lambda^{-1 / n} M .
$$

It follows that

$$
\left|\Omega_{M}\right| \leq C(n) R^{n} \leq C(n) \lambda^{-1 / 2} M^{n / 2} .
$$

Similarly, we have the following:

Proposition 2.11 Let $0<\Lambda<\infty$, and let $u$ be a convex viscosity solution of

$$
\operatorname{det}\left(D^{2} u\right) \leq \Lambda \text { on } \mathbb{R}^{n} .
$$

Assume that $u$ is normalized to further satisfy (2.6). Then there exists some constant $C \geq 1$, depending only only $n$, such that

$$
\left|\Omega_{M}\right| \geq C^{-1} \Lambda^{-1 / 2} M^{n / 2} \quad \forall M>0 .
$$

PROOF: In the proof of Proposition 2.10, we let instead

$$
w(x)=\Lambda^{-1 / n} u\left(A_{M}^{-1}(x)\right) .
$$

Then, by (2.7),

$$
\operatorname{det}\left(D^{2} w\right) \leq 1 \text { on } \mathbb{R}^{n} \quad \text { and } \quad w \geq \Lambda^{-1 / n} M \text { on } \partial B_{n R} .
$$

Applying Corollary 2.3, we have

$$
0=w\left(A_{M}(0)\right) \geq-\frac{1}{2}(n R)^{2}+\min _{\partial B_{n} R} w \geq-\frac{1}{2}(n R)^{2}+\Lambda^{-1 / n} M .
$$


It follows that

$$
\left|\Omega_{M}\right| \geq C(n)^{-1} R^{n} \geq C(n)^{-1} \Lambda^{-1 / 2} M^{n / 2}
$$

Proposition 2.12 For $0<\lambda \leq \Lambda<\infty$, let $u$ be a convex viscosity solution of (2.5) and (2.7) that also satisfies the normalization (2.6), and let $A_{M}(x)=$ $a_{M} x+b_{M}$ be an affine transformation satisfying (2.3) and (2.4). Then for some constant $C$, depending only on $n, \lambda$, and $\Lambda$,

$$
2 n R \geq \operatorname{dist}\left(A_{M}\left(\Omega_{M / 2}\right), \partial A_{M}\left(\Omega_{M}\right)\right) \geq C^{-1} R \quad \forall M>0 .
$$

Consequently,

$$
B_{R / C} \subset a_{M}\left(\Omega_{M}\right) \subset B_{2 n R}
$$

ProOF: Let $w$ be defined on $O_{M}:=\frac{1}{R} A_{M}\left(\Omega_{M}\right)$ by

$$
w(x)=\frac{\Lambda^{-1 / n}}{R^{2}} u\left(A_{M}^{-1}(R x)\right)-\frac{\Lambda^{-1 / n} M}{R^{2}}, \quad x \in O_{M} .
$$

Then

$$
B_{1} \subset O_{M} \subset B_{n}, \quad w=0 \text { on } O_{M},
$$

and

$$
\operatorname{det}\left(D^{2} w\right) \leq 1 \text { in } O_{M} .
$$

It follows from Lemma A. 1 that

$$
\left.w(x) \geq-C(n) \operatorname{dist}\left(x, \partial O_{M}\right)\right)^{\frac{2}{n+1}}, \quad x \in O_{M} .
$$

For $\bar{y} \in A_{M}\left(\Omega_{M / 2}\right), M>0$, let $\bar{x}=\frac{1}{R} \bar{y}$; we then have

$$
-\frac{\Lambda^{-1 / n} M}{2 R^{2}}=\frac{\Lambda^{-1 / n}\left(\frac{M}{2}-M\right)}{R^{2}} \geq w(\bar{x}) \geq-C(n) \operatorname{dist}\left(\bar{x}, \partial O_{M}\right)^{\frac{2}{n+1}} .
$$

It follows from Proposition 2.10, (2.3), and (2.4) that for some $C=C(n, \Lambda, \lambda) \geq 1$,

$$
\operatorname{dist}\left(\bar{y}, \partial A_{M}\left(\Omega_{M}\right)\right) \geq C^{-1} R \text {. }
$$

Since $A_{M}\left(\Omega_{M}\right) \subset B_{n R}$,

$$
\operatorname{dist}\left(\bar{y}, \partial A_{M}\left(\Omega_{M}\right)\right) \leq 2 n R .
$$

Estimate (2.8) is established. Estimate (2.9) follows from (2.8) and

$$
\operatorname{dist}\left(A_{M}(0), \partial A_{M}\left(\Omega_{M}\right)\right)=\operatorname{dist}\left(0, \partial a_{M}\left(\Omega_{M}\right)\right) .
$$

PROOF OF THEOREM 2.7: Without loss of generality, we may assume that $u$ further satisfies (2.6). For $M>0$, by Proposition 2.8, $\Omega_{M}$ is bounded and convex. Applying Proposition 2.4 to $w=u-M$ on $\Omega=\Omega_{M}$, we have $u \in C^{\infty}\left(\Omega_{M}\right)$. Since $\mathbb{R}^{n}=\cup_{M>0} \Omega_{M}$, we have $u \in C^{\infty}\left(\mathbb{R}^{n}\right)$. 
PROPOSITION 2.13 Let $u \in C^{2}\left(\mathbb{R}^{n}\right)$ be a convex solution of (2.7) that further satisfies normalizations (2.6) and

$$
D^{2} u(0)=I \text { the identity. }
$$

For $M>0$, let $A_{M}(x)=a_{M} x+b_{M}$ be some affine transformation satisfying (2.3) and (2.4). Then for some positive constant $C=C(n)$,

$$
C^{-1} M^{1 / 2} \leq R \leq C M^{1 / 2}
$$

and

$$
\left\|a_{M}\right\| \leq C, \quad\left\|a_{M}^{-1}\right\| \leq C
$$

Moreover,

$$
\sup _{\mathbb{R}^{n}}\left\|D^{2} u\right\| \leq C
$$

PROOF: Estimate (2.10) follows from Propositions 2.10 and 2.11 and (2.4). Consider

By (2.9),

$$
w(x)=\frac{1}{R^{2}} u\left(a_{M}^{-1}(R x)\right), \quad x \in O:=\frac{1}{R} a_{M}\left(\Omega_{M}\right) .
$$

$$
B_{1 / C} \subset O \subset B_{2 n}
$$

By (1.1),

$$
\operatorname{det}\left(D^{2} w\right)=1 \quad \text { on } O
$$

It follows from (2.10) that

$$
C^{-1} \leq w=\frac{M}{R^{2}} \leq C \quad \text { on } \partial O .
$$

By (2.13) and the interior second derivative estimates of Pogorelov,

$$
\left\|D^{2} w(x)\right\| \leq C \quad \forall|x| \leq \frac{1}{2 C},
$$

in particular,

$$
\left\|D^{2} w(0)\right\| \leq C .
$$

Recall the Pogorelov estimate: Let $O$ be a bounded convex open set of $\mathbb{R}^{n}$, and let $w \in C^{2}(O) \cap C^{0}(\bar{O})$ be a convex function satisfying $\operatorname{det}\left(D^{2} w\right)=1$ in $O$ and $w=0$ on $\partial$; then for any compact subset $K$ of $O,\left\|D^{2} w\right\| \leq C$ on $K$ for some constant $C$ depending only on $n, O$, and $K$.

Since $D^{2} u(0)=I$, we have

$$
D^{2} w(0)=\left(a_{M}^{-1}\right)^{\prime}\left(a_{M}^{-1}\right),
$$

where $\left(a_{M}^{-1}\right)^{\prime}$ denotes the transpose of $a_{M}^{-1}$. It follows that

$$
\left\|a_{M}^{-1}\right\| \leq C \text {. }
$$


Since $\operatorname{det}\left(a_{M}^{-1}\right)=\operatorname{det}\left(a_{M}\right)=1$, we then have

$$
\left\|a_{M}\right\| \leq C .
$$

Estimate (2.11) is established.

By (2.14) and (2.11),

$$
\sup _{|y| \leq \frac{R}{2 C}}\left|D^{2} u(y)\right| \leq C \quad \text { where } C=C(n) .
$$

Since $M$ can be arbitrary large (as can $R$ ), estimate (2.12) follows from the above.

Theorem 1.1 can be deduced from Theorem 2.7, (2.12), and the $C^{2, \alpha}$ interior estimates of Evans and Krylov as follows:

Proof of Theorem 1.1: By Theorem 2.7, $u \in C^{\infty}\left(\mathbb{R}^{n}\right)$. Then, by (2.12),

$$
|u(x)| \leq C|x|^{2}, \quad|x| \geq 1 .
$$

For $\bar{x} \in \mathbb{R}^{n}$, we will show that $D^{2} u(\bar{x})=D^{2} u(0)$. For $R>2(|\bar{x}|+1)$, we consider

$$
w(y)=\frac{1}{R^{2}} u(R y), \quad y \in B_{1} .
$$

By (2.15), (1.1), and (2.12),

$$
|w| \leq C, \quad \operatorname{det}\left(D^{2} w\right)=1, \quad\left|D^{2} w\right| \leq C \text { on } B_{1}
$$

where $C=C(n)$. Let

$$
\bar{y}=\frac{\bar{x}}{R} .
$$

Then

$$
|\bar{y}| \leq \frac{1}{2}
$$

It follows from the above-mentioned estimates of Evans and Krylov that for some $\alpha \in(0,1)$ and $C$ (independent of $R$ ),

$$
\left|D^{2} w(\bar{y})-D^{2} w(0)\right| \leq C|\bar{y}|^{\alpha},
$$

i.e.,

$$
\left|D^{2} u(\bar{x})-D^{2} u(0)\right| \leq C \frac{|\bar{x}|^{\alpha}}{R^{\alpha}} .
$$

Sending $R \rightarrow \infty$, we have

$$
D^{2} u(\bar{x})=D^{2} u(0) .
$$

Since $\bar{x}$ is arbitrary, $u$ is a quadratic polynomial. Theorem 1.1 is established. 


\section{Proof of Theorem 1.2 and Corollary 1.3}

We prove Theorem 1.2 and Corollary 1.3 in this section.

First we have the following:

LEMMA 3.1 Let $u$ be as in Theorem 1.2; then $u$ is $C^{\infty}$ in the complement of the support of $(f-1)$.

PROOF: Let $\bar{x}$ be in the complement of the support of $(f-1)$; without loss of generality, $\bar{x}=0$. Subtracting from $u$ a supporting plane to the graph of $u$ at $(0, u(0))$, we may assume without loss of generality that $u$ satisfies (2.6). It follows from Proposition 2.8 that $\Omega_{\epsilon}$ is bounded and convex for $\epsilon>0$. Then by [3, theorem 1] we have that $\left\{x \in \Omega_{\epsilon}: u(x)=0\right\}=\{0\}$. Taking $\epsilon>0$ small enough, $\Omega_{\epsilon}$ belongs to the complement of the support of $(f-1)$. It follows from Proposition 2.4 that $u \in C^{\infty}\left(\Omega_{\epsilon}\right)$.

Let $u$ be as in Theorem 1.2. As explained above, we may assume that $u$ also satisfies (2.6). We know from Lemma 3.1 that for $M$ large, $u \in C^{\infty}\left(\mathbb{R}^{n} \backslash \Omega_{M}\right)$ and $\Omega_{M}$ is bounded and strictly convex. Keep $u$ fixed outside $\Omega_{M}$ and redefine $u$ inside $\Omega_{M}$ so that the new $u$ is in $C^{\infty}\left(\mathbb{R}^{n}\right)$ and $\left(D^{2} u\right)>0$ on $\mathbb{R}^{n}$. Let $f=\operatorname{det}\left(D^{2} u\right)$ be the new $f$. So we only need to establish Theorem 1.2 with the additional hypothesis that $u \in C^{\infty}\left(\mathbb{R}^{n}\right)$ and $u$ satisfies (2.6). These will be assumed in the rest of this section.

Let $A_{M}(x)=a_{M} x+b_{M}$ be an affine transformation satisfying (2.3) and (2.4), and let

By (2.9),

$$
\xi(y)=\frac{1}{R^{2}} u\left(a_{M}^{-1}(R y)\right), \quad y \in O:=\frac{1}{R} a_{M}\left(\Omega_{M}\right) .
$$

$$
B_{1 / C} \subset O \subset B_{2 n}
$$

Here and in the following, $C \geq 1$ denotes some constant depending only on $n$ and $f$. Clearly

$$
\operatorname{det}\left(D^{2} \xi\right)=f\left(a_{M}^{-1}(R y)\right) .
$$

By Propositions 2.10 and 2.11,

$$
\xi=\frac{M}{R^{2}} \in\left(C^{-1}, C\right) \quad \text { on } \partial O
$$

By Proposition 2.4 and Remark 2.5, there exists a unique convex solution $\bar{\xi}:=$ $\bar{\xi}_{O, M / R^{2}} \in C^{0}(\bar{O}) \cap C^{\infty}(O)$ of

$$
\begin{cases}\operatorname{det}\left(D^{2} \bar{\xi}\right)=1 & \text { on } O \\ \bar{\xi}=\frac{M}{R^{2}} & \text { on } \partial O\end{cases}
$$

Because of (3.1), the interior second derivative estimates of Pogorelov, and the $C^{2, \alpha}$ estimates of Evans and Krylov (also use Schauder estimates), for every $\delta>0$, there exists some positive constant $C=C(\delta)$, independent of $M$, such that

$$
C^{-1} I \leq\left(D^{2} \bar{\xi}(x)\right) \leq C I, \quad\left|D^{3} \bar{\xi}(x)\right| \leq C, \quad x \in O, \operatorname{dist}(x, \partial O) \geq \delta .
$$


LEMMA 3.2 For some positive constant $C$ independent of $M$,

$$
|\xi-\bar{\xi}| \leq C R^{-1} \quad \text { in } O
$$

Proof: By the Alexandrov estimate (see, e.g., [16, lemma 9.2]),

$$
-\min _{\bar{O}}(\xi-\bar{\xi}) \leq C\left\{\int_{S^{+}} \operatorname{det}\left(D^{2}(\xi-\bar{\xi})\right\}^{1 / n},\right.
$$

where

$$
S^{+}=\left\{x \in O: D^{2}(\xi-\bar{\xi})(x)>0\right\}
$$

On $S^{+}$

$$
\frac{D^{2} \xi}{2}=\frac{D^{2}(\xi-\bar{\xi})+D^{2} \bar{\xi}}{2}
$$

so it follows from the concavity of (det $)^{1 / n}$,

$$
\operatorname{det}\left(\frac{D^{2} \xi}{2}\right)^{1 / n} \geq \frac{1}{2} \operatorname{det}\left(D^{2}(\xi-\bar{\xi})\right)^{1 / n}+\frac{1}{2} \operatorname{det}\left(D^{2} \bar{\xi}\right)^{1 / n}
$$

i.e.,

$$
\operatorname{det}\left(D^{2}(\xi-\bar{\xi})(y)\right)^{1 / n} \leq 1-f\left(a_{M}^{-1}(R y)\right)^{1 / n}, \quad y \in S^{+} .
$$

It follows that

$$
-\min _{\bar{O}}(\xi-\bar{\xi}) \leq C\left\{\int_{S^{+}}\left[1-f\left(a_{M}^{-1}(R y)\right)^{1 / n}\right]^{n}\right\}^{1 / n} \leq \frac{C}{R}\left\|1-f^{1 / n}\right\|_{L^{n}(f<1)} \leq \frac{C}{R} .
$$

Similarly, we can show that

$$
-\min _{\bar{O}}(\bar{\xi}-\xi) \leq \frac{C}{R}\left\|f^{1 / n}-1\right\|_{L^{n}(f>1)} \leq \frac{C}{R} .
$$

Lemma 3.2 is established.

Let $\bar{x}$ be the unique minimum point of $\bar{\xi}$ in $\bar{O}$. Recall that

$$
A_{M}(x)=a_{M} x+b_{M}
$$

is an affine transformation satisfying (2.3) and (2.4). By Proposition 2.10 and Proposition 2.11,

$$
C^{-1} M^{1 / 2} \leq R \leq C M^{1 / 2}
$$

Let

$$
E_{M}=\left\{x: x^{\prime} D^{2} \bar{\xi}(\bar{x}) x \leq 1\right\}
$$


PROPOSITION 3.3 There exist $\bar{k}$ and $C$, depending only on $n$ and $f$, such that for $\epsilon=\frac{1}{10}, M=2^{(1+\epsilon) k}$, and $2^{k-1} \leq M^{\prime} \leq 2^{k}$, we have

$$
\begin{aligned}
\left(\frac{2 M^{\prime}}{R^{2}}-C 2^{-\frac{3 \epsilon k}{2}}\right)^{1 / 2} E_{M} & \subset \frac{1}{R} a_{M}\left(\Omega_{M^{\prime}}\right) \\
& \subset\left(\frac{2 M^{\prime}}{R^{2}}+C 2^{-\frac{3 \epsilon k}{2}}\right)^{1 / 2} E_{M} \quad \forall k \geq \bar{k} .
\end{aligned}
$$

PROOF: In the proof, $C$ and $\bar{k}$ denote various large constants with the specified dependence, and we always assume that $k \geq \bar{k}$. Clearly,

$$
C^{-1} 2^{-\epsilon k} \leq \frac{M^{\prime}}{R^{2}} \leq C 2^{-\epsilon k}, \quad C^{-1} 2^{\frac{(1+\epsilon) k}{2}} \leq R \leq C 2^{\frac{(1+\epsilon) k}{2}}
$$

and

$$
\left\{\xi<\frac{M^{\prime}}{R^{2}}\right\}:=\left\{z \in O: \xi(z)<\frac{M^{\prime}}{R^{2}}\right\}=\frac{1}{R} a_{M}\left(\Omega_{M^{\prime}}\right) .
$$

By Lemma 3.2,

$$
|\xi-\bar{\xi}| \leq \frac{C}{R} \leq C 2^{-\frac{(1+\epsilon) k}{2}} \quad \text { on } \bar{O}
$$

Since

$$
\frac{M^{\prime}}{R^{2}} \ll \frac{C}{R},
$$

the level surface of $\xi$ can be well approximated by the level surface of $\bar{\xi}$ :

$$
\left\{\bar{\xi}<\frac{M^{\prime}}{R^{2}}-\frac{C}{R}\right\} \subset\left\{\xi<\frac{M^{\prime}}{R^{2}}\right\} \subset\left\{\bar{\xi}<\frac{M^{\prime}}{R^{2}}+\frac{C}{R}\right\} .
$$

By Lemma 3.2 and the fact $\xi \geq 0$, we have

$$
-\frac{C}{R} \leq \xi(\bar{x})-\frac{C}{R} \leq \bar{\xi}(\bar{x}) \leq \bar{\xi}(0) \leq \xi(0)+\frac{C}{R}=\frac{C}{R} .
$$

So, by Lemma A.1, $B_{1 / C}(\bar{x}) \subset O$, and therefore by (3.2),

$$
\left|\bar{\xi}(x)-\bar{\xi}(\bar{x})-\frac{1}{2}(x-\bar{x})^{\prime} D^{2} \bar{\xi}(\bar{x})(x-\bar{x})\right| \leq C|x-\bar{x}|^{3} \quad \forall x \in B_{1 / C}(\bar{x})
$$

and

$$
\frac{I}{C} \leq\left(D^{2} \bar{\xi}(\bar{x})\right) \leq C I
$$

Estimate (3.3) follows from (3.4) and the above estimates by elementary consideration.

Let $B$ denote the unit ball in $\mathbb{R}^{n}$, and still let $\epsilon=\frac{1}{10}$. 
PROPOSITION 3.4 There exist positive constants $C$ and $\bar{k}$ and some real invertible upper-triangular matrices $\left\{T_{k}\right\}_{k \geq \bar{k}}$ such that

$$
\operatorname{det}\left(T_{k}\right)=1, \quad\left\|T_{k} T_{k-1}^{-1}-I\right\| \leq C 2^{-\epsilon k},
$$

and

$$
\begin{array}{r}
\left(1-C 2^{-\epsilon k}\right) \sqrt{2 M^{\prime}} B \subset T_{k}\left(\Omega_{M^{\prime}}\right) \subset\left(1+C 2^{-\epsilon k}\right) \sqrt{2 M^{\prime}} B, \\
2^{k-1} \leq M^{\prime} \leq 2^{k} .
\end{array}
$$

Consequently, for some invertible $T$,

$$
\operatorname{det}(T)=1, \quad\left\|T_{k}-T\right\| \leq C 2^{-\epsilon k} .
$$

ProOF: Let $M=2^{(1+\epsilon) k}$ and let $2^{k-1} \leq M^{\prime} \leq 2^{k}$. By (3.3), there exist some constants $C$ and $\bar{k}$ (depending only on $n$ and $f$ ) such that

$$
\left(1-C 2^{-\epsilon k}\right) \sqrt{2 M^{\prime}} E_{M} \subset a_{M}\left(\Omega_{M^{\prime}}\right) \subset\left(1+C 2^{-\epsilon k}\right) \sqrt{2 M^{\prime}} E_{M}, \quad k \geq \bar{k} .
$$

Let $Q$ be the positive definite matrix satisfying $Q^{2}=D^{2} \bar{\xi}(\bar{x})$, and let $O$ be an orthogonal matrix such that

$$
T_{k}:=O Q a_{M} \text { is upper-triangular. }
$$

Clearly,

$$
\operatorname{det}\left(T_{k}\right)=\operatorname{det}(O) \operatorname{det}(Q) \operatorname{det}\left(a_{M}\right)=\sqrt{\operatorname{det}\left(D^{2} \bar{\xi}(\bar{x})\right)} \operatorname{det}\left(a_{M}\right)=1
$$

and

$$
\left(1-C 2^{-\epsilon k}\right) \sqrt{2 M^{\prime}} B \subset T_{k}\left(\Omega_{M^{\prime}}\right) \subset\left(1+C 2^{-\epsilon k}\right) \sqrt{2 M^{\prime}} B .
$$

Taking some larger $\bar{k}$, we deduce from (3.8), with $M^{\prime}=2^{k}$ and then $M^{\prime}=2^{k-1}$, that (with a larger $C$ )

$$
\left(1-C 2^{-\epsilon k}\right) B \subset T_{k} T_{k-1}^{-1}(B) \subset\left(1+C 2^{-\epsilon k}\right) B, \quad k \geq \bar{k} .
$$

Since $T_{k} T_{k-1}^{-1}$ is still upper-triangular, we apply Lemma A.5 (with $U=T_{k} T_{k-1}^{-1}$ ) to obtain that

$$
\left\|T_{k} T_{k-1}^{-1}-I\right\| \leq C 2^{-\epsilon k}, \quad k \geq \bar{k} .
$$

Estimates (3.5) and (3.6) are established. The existence of $T$ and (3.7) (with a larger $C$ ) follow by elementary consideration. Proposition 3.4 is established.

Let $v=u \circ T$. Then

$$
\operatorname{det}\left(D^{2} v\right)=1, \quad \mathbb{R}^{n} \backslash T^{-1}\left(\Omega_{M_{0}}\right) .
$$

Since $\left\{x: v(x)<M^{\prime}\right\}=T\left(\Omega_{M^{\prime}}\right)$, we deduce from (3.6) and (3.7) that $\left(1-C\left(M^{\prime}\right)^{-\epsilon}\right) \sqrt{2 M^{\prime}} B \subset\left\{x: v(x)<M^{\prime}\right\} \subset\left(1+C\left(M^{\prime}\right)^{-\epsilon}\right) \sqrt{2 M^{\prime}} B \quad \forall M^{\prime} \geq 2^{\bar{k}}$. 
Consequently,

$$
\left.\left.\left|v(x)-\frac{1}{2}\right| x\right|^{2}|\leq C| x\right|^{2-2 \epsilon}, \quad|x| \geq 2^{\bar{k}} .
$$

LEMMA 3.5 Let $w \in C^{\infty}\left(\mathbb{R}^{n} \backslash B_{1}\right)$ satisfy

$$
\left(I+D^{2} w(x)\right)>0, \quad \operatorname{det}\left(I+D^{2} w(x)\right)=1 \text { on }|x|>1,
$$

and, for some constants $\beta>0$ and $\gamma>-2$,

$$
|w(x)| \leq \frac{\beta}{|x|^{\gamma}} \quad \text { on }|x|>1 .
$$

Then there exist some constants $r=r(n, \beta, \gamma) \geq 1$ such that for all $k \geq 1$,

$$
\left|D^{k} w(x)\right| \leq \frac{C}{|x|^{\gamma+k}} \quad \text { on }|x| \geq r,
$$

where $C$ depends only on $n, k, \beta$, and $\gamma$.

PROOF: Let

$$
\eta(x):=\frac{|x|^{2}}{2}+w(x)
$$

For $|x|=R>8$, let

$$
\eta_{R}(y):=\left(\frac{4}{R}\right)^{2} \eta\left(x+\frac{R}{4} y\right), \quad|y| \leq 2,
$$

and

$$
w_{R}(y):=\left(\frac{4}{R}\right)^{2} w\left(x+\frac{R}{4} y\right)=\eta_{R}(y)-8\left|\frac{x}{R}+\frac{y}{4}\right|^{2}, \quad|y| \leq 2 .
$$

By the decay hypothesis on $w$, there exists some $r=r(n, \beta) \geq 1$ such that for $|x|=R \geq r$,

$$
3 \leq 4-\frac{16 \beta 2^{\gamma}}{R^{\gamma+2}} \leq \eta_{R}(y) \leq 32+\frac{16 \beta 2^{\gamma}}{R^{\gamma+2}} \leq 33, \quad|y| \leq 2 .
$$

Since $\eta_{R}$ satisfies

$$
\left(D^{2} \eta_{R}(y)\right)>0, \quad \operatorname{det}\left(D^{2} \eta_{R}(y)\right)=1, \quad|y|<2,
$$

by the estimates of Pogorelov, Evans-Krylov, and Schauder, we have, for every $k \geq 1$,

$$
\left\|\eta_{R}\right\|_{C^{k}(\bar{B})} \leq C \quad \text { and } \quad \frac{I}{C}<\left(D^{2} \eta_{R}\right)<C I \text { on } B .
$$

Here and in the following, $C \geq 1$ denotes some constant depending on $n$ and $k$ unless otherwise stated.

It follows that

$$
\left\|w_{R}\right\|_{C^{k}(\bar{B})} \leq C \quad \text { and } \quad \frac{I}{C}<\left(I+D^{2} w_{R}\right)<C I \text { on } B .
$$

Clearly, $w_{R}$ satisfies

$$
\hat{a}_{i j}(y) D_{i j} w_{R}(y)=0 \quad \text { on } B_{2}
$$


where $\left(\hat{a}_{i j}(y)\right)=\int_{0}^{1} F_{\xi_{i j}}\left(I+s D^{2} w_{R}(y)\right) d s$ satisfies, in view of (3.11), that

$$
\left\|\hat{a}_{i j}\right\|_{C^{k}(\bar{B})} \leq C \text { and } \frac{I}{C}<\left(\hat{a}_{i j}\right)<C I \text { on } B \text {. }
$$

Here and throughout the section, we use the notation

$$
F(\xi):=\operatorname{det}\left(\xi_{i j}\right)^{1 / n}
$$

It is well-known that, in the open set of symmetric positive definite matrices, $\left(F_{\xi_{i j}}\right)$ is positive definite and $F$ is concave.

By Schauder theory,

$$
\left|D^{k} w_{R}(0)\right| \leq C\left\|w_{R}\right\|_{L^{\infty}(B)} \leq \frac{C(n, k, \beta, \gamma)}{R^{\gamma+2}} .
$$

It follows that

$$
\left|D^{k} w(x)\right| \leq \frac{C(n, k, \beta, \gamma)}{|x|^{\gamma+k}}
$$

LEMMA 3.6 For $n \geq 3$, there exist $b \in \mathbb{R}^{n}, c \in \mathbb{R}$, and some positive constant $C$ such that

$$
\left|v(x)-\left(\frac{|x|^{2}}{2}+b \cdot x+c\right)\right| \leq \frac{C}{|x|^{n-2}} \quad \forall x \in \mathbb{R}^{n} \backslash T^{-1}\left(\Omega_{M_{0}}\right) .
$$

PROOF: Let

$$
\hat{E}:=v(x)-\frac{|x|^{2}}{2}
$$

Then

$$
F\left(I+D^{2} \hat{E}\right)=1 \quad \text { in } \mathbb{R}^{n} \backslash T^{-1}\left(\Omega_{M_{0}}\right) .
$$

By (3.10), we apply Lemma 3.5 to $\hat{E}$ (rather, to $r^{-2} \hat{E}(r x)$, for some harmless $r$ ) with $\gamma=2 \epsilon-2$ to obtain

It follows that

$$
\left|D^{2} \hat{E}(x)\right| \leq \frac{C}{|x|^{2 \epsilon}}
$$

$$
\tilde{a}_{i j}(x) D_{i j} \hat{E}(x)=F\left(I+D^{2} \hat{E}\right)-F(I)=0 \quad \text { in } \mathbb{R}^{n} \backslash T^{-1}\left(\Omega_{M_{0}}\right),
$$

where

$$
\tilde{a}_{i j}(x)=\int_{0}^{1} F_{\xi_{i j}}\left(I+s D^{2} \hat{E}(x)\right) d s .
$$

Let $e \in \mathbb{R}^{n}$ be a unit vector; applying $D_{e}$ and $D_{e e}$ to (3.12) yields, in view of the concavity of $F(\xi)$, that

$$
a_{i j}(x) D_{i j}\left(D_{e} \hat{E}(x)\right)=0, \quad x \in \mathbb{R}^{n} \backslash T^{-1}\left(\Omega_{M_{0}}\right),
$$

and

$$
a_{i j}(x) D_{i j}\left(D_{e e} \hat{E}(x)\right) \geq 0, \quad x \in \mathbb{R}^{n} \backslash T^{-1}\left(\Omega_{M_{0}}\right),
$$


where

$$
a_{i j}(x):=F_{\xi_{i j}}\left(I+D^{2} \hat{E}(x)\right) .
$$

Clearly, $\left(a_{i j}(x)\right)$ and $\left(\tilde{a}_{i j}(x)\right)$ are positive definite and satisfy

$$
\left|\tilde{a}_{i j}(x)-\delta_{i j}\right|+\left|a_{i j}(x)-\delta_{i j}\right| \leq C|x|^{-2 \epsilon} .
$$

It is well-known that for such coefficients, there exist positive solutions $G(x)$ of $a_{i j}(x) D_{i j} G(x)=0$ satisfying $\lim _{|x| \rightarrow \infty}\left(|x|^{n-2} G(x)\right)=1$. By (3.15) and the maximum principle,

$$
e^{\prime} D^{2} \hat{E}(x) e=D_{e e} \hat{E}(x) \leq C G(x) \leq C|x|^{2-n}, \quad x \in T^{-1}\left(\Omega_{M_{0}}\right) .
$$

This means that the largest eigenvalue of $\left(D^{2} \hat{E}(x)\right)$ is bounded from above by $C|x|^{2-n}$. By (3.13), the least eigenvalue of $\left(D^{2} \hat{E}(x)\right)$ is bounded below by a negative constant multiple (depending only on the ellipticity of $\left.\left(\tilde{a}_{i j}(x)\right)\right)$ of the largest eigenvalue of $\left(D^{2} \hat{E}(x)\right)$. Thus,

$$
e^{\prime} D^{2} \hat{E}(x) e=D_{e e} \hat{E}(x) \geq-C|x|^{2-n} .
$$

It follows that

$$
\left|D^{2} \hat{E}(x)\right| \leq \frac{C}{|x|^{n-2}} \leq \frac{C}{|x|}, \quad \mathbb{R}^{n} \backslash T^{-1}\left(\Omega_{M_{0}}\right) .
$$

For $1 \leq m \leq n, D_{m} \hat{E}$ satisfies (3.14) with $D_{e} \hat{E}$ replaced by $D_{m} \hat{E}$. Applying theorem 4 in [15] (with $u=D_{m} \hat{E}$ and $r \rightarrow \infty$ ), $D_{m} \hat{E}(x)$ tends to some constant, denoted as $b_{m}$, as $|x|$ tends to infinity, namely,

$$
\lim _{|x| \rightarrow \infty} D \widetilde{E}(x)=0 \quad \text { where } \quad \widetilde{E}(x):=\hat{E}(x)-b \cdot x .
$$

Since $D_{m} \widetilde{E}$ satisfies the same equation as $D_{m} \hat{E}$ (i.e., (3.14)), we deduce from the maximum principle that

$$
|D \widetilde{E}(x)| \leq C|x|^{2-n} \leq \frac{C}{|x|}, \quad \mathbb{R}^{n} \backslash T^{-1}\left(\Omega_{M_{0}}\right) .
$$

By (3.13),

$$
\widetilde{a}_{i j}(x) D_{i j} \widetilde{E}(x)=0, \quad \mathbb{R}^{n} \backslash T^{-1}\left(\Omega_{M_{0}}\right) .
$$

Applying again [15, theorem 4] to $u=\widetilde{E}$ as $r \rightarrow \infty$, we have

$$
\lim _{|x| \rightarrow \infty} \widetilde{E}(x)=c
$$

for some constant $c$. Applying the maximum principle to $\widetilde{E}(x)-c$, we have

$$
|\widetilde{E}(x)-c| \leq C|x|^{2-n},
$$

namely,

$$
|\hat{E}(x)-b \cdot x-c| \leq C|x|^{2-n}
$$




\subsection{Proof of Theorem 1.2}

In this subsection we complete the proof of Theorem 1.2.

For $n \geq 3$, inequality (1.6) follows from Lemma 3.6, and estimate (1.7) follows from (1.6) and Lemma 3.5 with $\gamma=n-2$. This completes the proof of Theorem 1.2 in the case $n \geq 3$.

In the rest of this subsection we prove Theorem 1.2 when $n=2$. First, instead of Lemma 3.6, we have the following:

LEMMA 3.7 For $n=2$, there exist $b \in \mathbb{R}^{n}, c, d \in \mathbb{R}$, and some positive constant C such that

$$
\left|v(x)-\left(\frac{|x|^{2}}{2}+b \cdot x+d \log |x|+c\right)\right| \leq \frac{C}{|x|} \quad \forall x \in \mathbb{R}^{n} \backslash T^{-1}\left(\Omega_{M_{0}}\right) .
$$

ProOF: By (3.10) and Lemma 3.5, we have

$$
|x|^{2 \epsilon-1}|\nabla \hat{E}(x)|+|x|^{2 \epsilon}\left|\nabla^{2} \hat{E}(x)\right|+|x|^{1+2 \epsilon}\left|\nabla^{3} \hat{E}(x)\right| \leq C, \quad|x| \geq 2^{\bar{k}},
$$

where

$$
\hat{E}(x):=v(x)-\frac{|x|^{2}}{2} .
$$

Differentiating (3.9) and using the above decay estimates on $D^{2} \hat{E}$, we have

$$
\left(\delta_{i j}+O\left(\left|D^{2} \hat{E}\right|\right) D_{i j}\left(\hat{E}_{m}\right)=0, \quad \text { where } \quad \hat{E}_{m}=\frac{\partial \hat{E}}{\partial x_{m}}, \quad m=1,2 .\right.
$$

It follows that

$$
\Delta \hat{E}_{m}=O\left(\left|D^{2} \hat{E}\right|\left|D^{3} \hat{E}\right|\right)=O\left(\frac{1}{|x|^{1+4 \epsilon}}\right) .
$$

Let

$$
\hat{\psi}_{m}(x)=-\frac{1}{2 \pi} \int_{|y| \geq 2^{\bar{k}}} \Delta \hat{E}_{m}(y)(\log |x-y|-\log |y|) d y .
$$

Then

$$
\Delta\left(\hat{E}_{m}-\hat{\psi}_{m}\right)=0, \quad|x| \geq 2^{\bar{k}},
$$

and, for any $\epsilon^{\prime}<2 \epsilon$,

$$
\left|\hat{\psi}_{m}(x)\right| \leq C\left(\epsilon^{\prime}\right)|x|^{1-\epsilon^{\prime}}, \quad|x| \geq 2^{\bar{k}} .
$$

Since $\hat{E}_{m}-\hat{\psi}_{m}$ is harmonic in $|x|>2^{\bar{k}}$, and its growth is at most of order $|x|^{1-2 \epsilon}$,

$$
\hat{E}_{m}(x)-\hat{\psi}_{m}(x)=O(\log |x|),
$$

and therefore, for any $\epsilon^{\prime}<4 \epsilon$,

$$
|\nabla \hat{E}(x)| \leq C\left(\epsilon^{\prime}\right)\left(\log |x|+|x|^{1-\epsilon^{\prime}}\right) .
$$

Integrating the above, we have, for any $\epsilon^{\prime}<4 \epsilon$,

$$
|\hat{E}(x)| \leq C\left(\epsilon^{\prime}\right)\left(|x| \log |x|+|x|^{2-\epsilon^{\prime}}\right) .
$$


We have improved estimate (3.10) of $|\hat{E}(x)|$. Applying Lemma 3.5 with this improved estimate and arguing as above, we have, for any $\epsilon^{\prime}<8 \epsilon$,

$$
|\hat{E}(x)| \leq C\left(\epsilon^{\prime}\right)\left(|x| \log |x|+|x|^{2-\epsilon^{\prime}}\right) .
$$

By induction, we have, for any $\epsilon^{\prime}>0$,

$$
|\hat{E}(x)| \leq C\left(\epsilon^{\prime}\right)|x|^{1+\epsilon^{\prime}}, \quad|x| \geq 2^{\bar{k}} .
$$

Then by Lemma 3.5, we have, for any $\epsilon^{\prime}>0$,

$$
|x|^{-\epsilon^{\prime}}|\nabla \hat{E}(x)|+|x|^{1-\epsilon^{\prime}}\left|\nabla^{2} \hat{E}(x)\right|+|x|^{2-\epsilon^{\prime}}\left|\nabla^{3} \hat{E}(x)\right| \leq C, \quad|x| \geq 2^{\bar{k}} .
$$

Since $\hat{E}$ satisfies

$$
\delta_{i j}+O\left(\left|D^{2} \hat{E}\right|\right) D_{i j} \hat{E}=0
$$

we have, for any $\epsilon^{\prime}>0$,

$$
\Delta \hat{E}=O\left(\left|D^{2} \hat{E}\right|^{2}\right)=O\left(\frac{1}{|x|^{4-\epsilon^{\prime}}}\right)
$$

Let

$$
\hat{\psi}(x)=-\frac{1}{2 \pi} \int_{|y| \geq 2^{\bar{k}}} \Delta \hat{E}(y)(\log |x-y|-\log |y|) d y .
$$

Then, for any $\epsilon^{\prime}>0$,

$$
|\hat{\psi}(x)| \leq \frac{C\left(\epsilon^{\prime}\right)}{|x|^{2-\epsilon^{\prime}}}, \quad|x| \geq 2^{\bar{k}} .
$$

Since $\hat{E}-\hat{\psi}$ is harmonic in $|x|>2^{\bar{k}}$, and since its growth is at most of order $|x|^{1+\epsilon^{\prime}}$, we have, for some $b \in \mathbb{R}^{n}$ and $c, d \in \mathbb{R}$, that

$$
\hat{E}(x)-\psi(x)=b \cdot x+d \log |x|+c+O\left(\frac{1}{|x|}\right) .
$$

By the decay of $\psi$,

$$
\hat{E}(x)=b \cdot x+d \log |x|+c+O\left(\frac{1}{|x|}\right) .
$$

Next we have the following proof:

PROOF OF THEOREM 1.2 FOR $n=2$ : Relation (1.8) follows from Lemma 3.7, and estimate (1.10), for some $d \in \mathbb{R}$, follows from (1.8) and Lemma 3.5 with $\gamma=1$. We only need to establish (1.9). In fact, by making an affine transformation, we only need to establish (1.9) for $A=I$. We first prove it under an additional hypothesis that $f \in C^{\infty}\left(\mathbb{R}^{2}\right)$. In this case $u \in C^{\infty}\left(\mathbb{R}^{2}\right)$. Write $w=|x|^{2} / 2$, $\eta=d \log |x|, \bar{u}=w+\eta+E$, and

$$
\operatorname{det}\left(D^{2} \bar{u}\right)=\bar{u}_{11} \bar{u}_{22}-\bar{u}_{12}^{2}=\partial_{1}\left(\bar{u}_{1} \bar{u}_{22}\right)-\partial_{2}\left(\bar{u}_{1} \bar{u}_{12}\right) .
$$


By (1.3) and $u=\bar{u}+b \cdot x+c$,

$$
\operatorname{det}\left(D^{2} \bar{u}\right)=\operatorname{det}\left(D^{2} u\right)=f
$$

By (1.10), as $|x| \rightarrow \infty$,

$$
|D E(x)|=O\left(\frac{1}{|x|^{2}}\right) \text { and }\left|D^{2} E(x)\right|=O\left(\frac{1}{|x|^{3}}\right) .
$$

Integrating the equation of $\bar{u}$ on $B_{r}$ and integrating by parts, we have, as $r \rightarrow \infty$,

$$
\begin{aligned}
\int_{B_{r}} f= & \int_{|x|=r}\left[\left(\bar{u}_{1} \bar{u}_{22}\right) \frac{x_{1}}{|x|}-\left(\bar{u}_{1} \bar{u}_{12}\right) \frac{x_{2}}{|x|}\right] \\
= & \int_{|x|=r}\left[\left(w_{1} w_{22}\right) \frac{x_{1}}{|x|}-\left(w_{1} w_{12}\right) \frac{x_{2}}{|x|}\right]+\int_{|x|=r}\left[\left(\eta_{1} w_{22}\right) \frac{x_{1}}{|x|}-\left(\eta_{1} w_{12}\right) \frac{x_{2}}{|x|}\right] \\
& +\int_{|x|=r}\left[\left(w_{1} \eta_{22}\right) \frac{x_{1}}{|x|}-\left(w_{1} \eta_{12}\right) \frac{x_{2}}{|x|}\right]+O\left(\frac{1}{r}\right) \\
= & \int_{B_{r}} \operatorname{det}\left(D^{2} w\right)+\int_{|x|=r}\left(\left[\eta_{1} w_{22}+w_{1} \eta_{22}\right] \frac{x_{1}}{|x|}-\left(\eta_{1} w_{12}\right) \frac{x_{2}}{|x|}\right)+O\left(\frac{1}{r}\right) .
\end{aligned}
$$

We know that

$$
\int_{B_{r}} \operatorname{det}\left(D^{2} w\right)=\pi r^{2}
$$

and

$$
\begin{aligned}
\int_{|x|=r}\left(\left[\eta_{1} w_{22}+w_{1} \eta_{22}\right] \frac{x_{1}}{|x|}-\left(\eta_{1} w_{12}\right) \frac{x_{2}}{|x|}\right) & =\frac{2 d}{r^{3}} \int_{|x|=r}\left(x_{1}^{2}\right) \\
& =\frac{d}{r^{3}} \int_{|x|=r}|x|^{2}=2 \pi d .
\end{aligned}
$$

It follows that

$$
\int_{B_{r}}(f-1)=2 \pi d+O\left(\frac{1}{r}\right) \quad \text { as } r \rightarrow \infty .
$$

Formula (1.9) for $A=I$ follows after sending $r$ to infinity. As pointed out earlier, formula (1.9) in general follows by applying the special case to $\tilde{u}(x)=u\left(A^{-1 / 2} x\right)$.

If $f \in C^{0}$ only, let $\bar{u}_{\epsilon}=\bar{u} * \rho_{\epsilon}$, the convolution of $\bar{u}$ and $\rho_{\epsilon}$ with $\rho_{\epsilon}(x)=$ $\epsilon^{-2} \rho\left(\epsilon^{-1} x\right)$, where $\rho$ is some nonnegative smooth function of compact support satisfying $\int \rho=1$. For large $r, B_{r}$ contains the support of $(f-1)$, and therefore $\bar{u}$ is $C^{\infty}$ near $\partial B_{r}$. We also know that

$$
\lim _{\epsilon \rightarrow 0} \int_{B_{r}} \operatorname{det}\left(D^{2} \bar{u}_{\epsilon}\right)=\int_{B_{r}} f .
$$


As shown above,

$$
\int_{B_{r}} \operatorname{det}\left(D^{2} \bar{u}_{\epsilon}\right)=\int_{|x|=r}\left[\left(\bar{u}_{\epsilon 1} \bar{u}_{\epsilon 22}\right) \frac{x_{1}}{|x|}-\left(\bar{u}_{\epsilon 1} \bar{u}_{\epsilon 12}\right) \frac{x_{2}}{|x|}\right] .
$$

Sending $\epsilon$ to zero, we have

$$
\int_{B_{r}} f=\int_{|x|=r}\left[\left(\bar{u}_{1} \bar{u}_{22}\right) \frac{x_{1}}{|x|}-\left(\bar{u}_{1} \bar{u}_{12}\right) \frac{x_{2}}{|x|}\right] .
$$

Following previous arguments, we obtain (1.9) (for continuous $f$ ).

\subsection{Proof of Corollary 1.3}

Finally, we can prove Corollary 1.3.

By enlarging $O$ slightly, we may assume that $u \in C^{0}\left(\mathbb{R}^{n} \backslash O\right)$. We divide the proof into three steps.

Step 1. First we prove Corollary 1.3 under the additional hypothesis that $u$ can be extended as a convex function on $\mathbb{R}^{n}$.

In this case, we first show that

$$
u \in C^{\infty}\left(\mathbb{R}^{n} \backslash \bar{O}\right) .
$$

For $\bar{x} \in \mathbb{R}^{n} \backslash \bar{O}$, by subtracting from $u$ a supporting plane to the graph at $(\bar{x}, u(\bar{x}))$, we may assume that $u \geq 0$ on $\mathbb{R}^{n} \backslash \bar{O}$ and $u(\bar{x})=0$. An application of Lemma 2.9 yields that $u(x) \rightarrow \infty$ as $|x| \rightarrow \infty$. For large $M,\{u<M\}$ contains $\bar{x}$ and $\bar{O}$. Applying theorem 1 in [3] on $\{u<M\} \backslash \bar{O}$, we know that $\bar{x}$ is the only point in $\{u<M\} \backslash \bar{O}$ where $u=0$. So for $\epsilon>0$ small, $\{u<\epsilon\}$ is a convex open subset of $\mathbb{R}^{n} \backslash \bar{O}$. By Proposition 2.4 and Remark 2.5, $u$ is $C^{\infty}$ in $\{u<\epsilon\}$. In particular, $u$ is $C^{\infty}$ near $\bar{x}$. (3.16) is established.

For large $M,\{u<M\}$ is a strictly convex, bounded open set containing $\bar{O}$. As explained at the beginning of Section 3, we can keep $u$ fixed outside $\{u<M\}$ while redefining $u$ inside $\{u<M\}$ so that the new $u$ is in $C^{\infty}\left(\mathbb{R}^{n}\right)$ and $\left(D^{2} u\right)>0$ on $\mathbb{R}^{n}$. Let $f=\operatorname{det}\left(D^{2} u\right)$; Corollary 1.3 follows from Theorem 1.2 applied to the new $u$.

Step 2. We show that there exists some affine function $l(x)$ such that

$$
u(x)-l(x) \geq 0 \quad \text { in } \mathbb{R}^{n} \backslash \bar{O} .
$$

Without loss of generality, we may assume that $\bar{O} \subset B_{1}$. Fix some $\lambda \gg 1$; by subtracting from $u$ a supporting plane to the graph at $\left(-\lambda e_{1}, u\left(-\lambda e_{1}\right)\right)$, we may assume that $u \geq 0$ on $\mathbb{R}^{n} \backslash \Gamma$, where $\Gamma$ denotes the cone generated by $-\lambda e_{1}$ and $B_{1}$. Fix some $M>0$ such that

$$
u(x)<M, \quad x \in B_{3} \backslash O .
$$

By Lemma 2.9, $\lim _{\alpha \rightarrow \infty} u\left(-\alpha e_{2}\right)=\infty$. Let $\alpha$ be the largest value such that $u\left(-\alpha e_{2}\right) \leq M$. Then $3 \leq \alpha<\infty$ and $u\left(-\alpha e_{2}\right)=M$. Let $\Gamma_{i}$ denote the closed cone generated by $-\alpha e_{2}$ and $B_{i}, i=1,2$, and let $l_{1}(x)$ denote a supporting plane 
of the graph of $u$ at $\left(-\alpha e_{2}, u\left(-\alpha e_{2}\right)\right)$. Then $l_{1}\left(-\alpha e_{2}\right)=M$ and $u(x) \geq l_{1}(x)$ for $x \in \mathbb{R}^{n} \backslash \Gamma_{1}$. Since $u \leq M$ on $B_{2} \backslash O$, we know that $l_{1}(x) \leq M$ on $\Gamma_{2}$. Since $\lambda$ is large, $\Gamma_{2} \cap \Gamma$ is compact. By Lemma 2.9,

$$
\lim _{x \in \Gamma_{1},|x| \rightarrow \infty} u(x)=\infty .
$$

It follows that

$$
u(x) \geq l_{1}(x) \text { on } \mathbb{R}^{n} \backslash\{\text { a compact set }\} .
$$

Therefore (3.17) holds with $l(x)=l_{1}(x)-c_{2}$ for a suitably large $c_{2}$.

Step 3. Now we can complete the proof of Corollary 1.3.

By step 2, we may assume without loss of generality that $u \geq 0$ on $\mathbb{R}^{n} \backslash O$. By Lemma 2.9, $\lim _{|x| \rightarrow \infty} u(x)=\infty$. It follows that, for large $M$, the function

$$
\tilde{u}(x)= \begin{cases}M, & x \in \bar{O}, \\ \max \{M, u(x)\}, & x \in \mathbb{R}^{n} \backslash \bar{O},\end{cases}
$$

is convex on $\mathbb{R}^{n}$. Corollary 1.3(i) and Corollary 1.3(ii) follow from step 2 applied to $\tilde{u}$. In the following we show that $u \in C^{\infty}\left(\mathbb{R}^{n} \backslash \bar{O}\right)$. For $\bar{x} \in \mathbb{R}^{n} \backslash \bar{O}$, let $l(x)$ be a supporting plane to the graph of $u$ at $(\bar{x}, u(\bar{x}))$. We already know that $u$ grows quadratically at infinity. So, by applying [3, theorem 1], we know that $\bar{x}$ is an isolated local minimum point of $u(x)-l(x)$. By the same argument as in step $1, u$ is $C^{\infty}$ near $\bar{x}$.

\section{Proof of Theorem 1.7}

The uniqueness part in the proof of 1.7 can be deduced easily from the maximum principle (see, e.g., Lemma A.2). By the affine invariance of the problem, we may assume $A=I, b=0$, and $c=0$. We may also assume that

$$
\text { support of }(f-1) \subset B_{1} \text {. }
$$

First, we explore the proof under the additional hypothesis that $f \in C^{\infty}\left(\mathbb{R}^{n}\right)$. For $R>1$, let $u_{R} \in C^{\infty}\left(\bar{B}_{R}\right)$ be the unique convex solution of

$$
\begin{cases}\operatorname{det}\left(D^{2} u_{R}\right)=f & \text { on } B_{R} \\ u_{R}=\frac{R^{2}}{2} & \text { on } \partial B_{R} .\end{cases}
$$

We will show that as $R$ tends to infinity, $u_{R}$ tends to some $u$ that satisfies (1.3) and, for some constant depending only on $n, \min _{\mathbb{R}^{n}} f$ and $\max _{\mathbb{R}^{n}} f$.

$$
\sup _{\mathbb{R}^{n}}\left|u(x)-\frac{|x|^{2}}{2}\right| \leq C .
$$

To prove the above, we need some barrier functions. Let $h(r)$ be defined on $r>0$, and let

$$
u(x)=h(|x|)
$$


Then

So,

$$
D^{2} u(x)=\left(\begin{array}{llll}
h^{\prime \prime}(r) & & & \\
& \frac{h^{\prime}(r)}{r} & & \\
& & \ddots & \\
& & & \frac{h^{\prime}(r)}{r}
\end{array}\right)
$$

For $a=2^{n}\left(\max _{\mathbb{R}^{n}} f\right)$, let

$$
\operatorname{det}\left(D^{2} u(x)\right)=h^{\prime \prime}(r)\left(\frac{h^{\prime}(r)}{r}\right)^{n-1} .
$$

$$
h_{-}(r)= \begin{cases}\int_{1}^{r}\left(s^{n}+a\right)^{1 / n} d s, & r \geq 1, \\ \left(\max _{\mathbb{R}^{n}} f\right)^{1 / n}\left(r^{2}-1\right), & 0 \leq r<1,\end{cases}
$$

and

$$
u_{-}(x)=h_{-}(|x|), \quad x \in \mathbb{R}^{n} .
$$

Then $u_{-} \in C^{0}\left(\mathbb{R}^{n}\right) \cap C^{\infty}\left(\bar{B}_{1}\right) \cap C^{\infty}\left(\overline{\mathbb{R}^{n} \backslash B_{1}}\right), u_{-}$is locally convex in $\mathbb{R}^{n} \backslash B_{1}$,

$$
\begin{array}{ll}
\operatorname{det}\left(D^{2} u_{-}\right)=1 & \text { on } \mathbb{R}^{n} \backslash \bar{B}_{1}, \\
\operatorname{det}\left(D^{2} u_{-}\right)>f & \text { on } B_{1},
\end{array}
$$

and

$$
\lim _{r \rightarrow 1^{-}} h_{-}^{\prime}(r)<\lim _{r \rightarrow 1^{+}} h_{-}^{\prime}(r) .
$$

It is easy to see that (recall that $n \geq 3$ )

$$
\sup _{x \in \mathbb{R}^{n}}\left|u_{-}(x)-\frac{|x|^{2}}{2}\right|<\infty \text {. }
$$

Next we define

$$
h_{+}(r)=\int_{1}^{r}\left(s^{n}-1\right)^{1 / n} d s, \quad r \geq 1
$$

and

$$
u_{+}(x)= \begin{cases}h_{+}(|x|), & |x| \geq 1 \\ 0, & |x|<1\end{cases}
$$

Then $u_{+} \in C^{0}\left(\mathbb{R}^{n}\right) \cap C^{1}\left(\bar{B}_{1}\right) \cap C^{\infty}\left(\mathbb{R}^{n} \backslash \bar{B}_{1}\right), u_{+}$is locally convex in $\mathbb{R}^{n} \backslash B_{1}$, and

$$
\operatorname{det}\left(D^{2} u_{+}\right)=1 \quad \text { on } \mathbb{R}^{n} \backslash \bar{B}_{1} \text {. }
$$

We also know that

$$
h_{+}^{\prime}(1)=0
$$

and

$$
\sup _{x \in \mathbb{R}^{n}}\left|u_{+}(x)-\frac{|x|^{2}}{2}\right|<\infty
$$


By (4.4) and (4.6),

$$
\beta_{+}:=\sup _{x \in \mathbb{R}^{n}}\left(\frac{|x|^{2}}{2}-u_{+}(x)\right)<\infty
$$

and

$$
\beta_{-}:=\inf _{x \in \mathbb{R}^{n}}\left(\frac{|x|^{2}}{2}-u_{-}(x)\right)>-\infty .
$$

We will use $\left(u_{+}+\beta_{+}\right)$and $\left(u_{-}+\beta_{-}\right)$as barrier functions to establish the following:

LEMMA 4.1 Let $u_{R}$ be defined in (4.1) for $R>1$; then

$$
u_{-}(x)+\beta_{-} \leq u_{R}(x) \leq u_{+}(x)+\beta_{+} \quad \forall x \in B_{R} .
$$

PROOF: Let $R>1$; for $\beta$ sufficiently large, we have

$$
u_{+}(x)+\beta \geq u_{R}(x), \quad x \in \bar{B}_{R} .
$$

Let $\bar{\beta}$ be the smallest number for which the above holds with $\beta=\bar{\beta}$. If $\bar{\beta} \leq \beta_{+}$, then the second inequality in (4.7) holds. Otherwise, $\bar{\beta}>\beta_{+}$, and for some $\bar{x} \in$ $\bar{B}_{R}$,

$$
u_{R}(\bar{x})=u_{+}(\bar{x})+\bar{\beta} .
$$

In view of the boundary data of $u_{R}$ and the definition of $\beta_{+}$(recall that $\bar{\beta}>\beta_{+}$), we must have

$$
|\bar{x}|<R
$$

We also know that

$$
|\bar{x}|>1 \text {. }
$$

Indeed, if $|\bar{x}| \leq 1$, the supporting plane of the graph of $u_{R}$ at $\left(\bar{x}, u_{R}(\bar{x})\right)$ must be horizontal ((4.5) is used when $|\bar{x}|=1)$, and therefore $u_{R}(x)=\bar{\beta}$ for all $|x| \leq 1$, which is impossible.

On the other hand, by the strong maximum principle, $1<|\bar{x}|<R$ cannot occur either. We have established the second inequality in (4.7).

To establish the first inequality, we argue similarly, using $\left(u_{-}+\beta_{-}\right)$as a barrier function. First, we know that for $\beta$ very negative, we have

$$
u_{-}(x)+\beta \leq u_{R}(x), \quad x \in \bar{B}_{R} .
$$

Let $\bar{\beta}$ be the largest number for which the above holds with $\beta=\bar{\beta}$. If $\bar{\beta} \geq \beta_{-}$, then the first inequality in (4.7) holds. Otherwise, $\bar{\beta}<\beta_{-}$, and for some $\bar{x} \in \bar{B}_{R}$,

$$
u_{R}(\bar{x})=u_{-}(\bar{x})+\bar{\beta} .
$$

Since

$$
\operatorname{det}\left(D^{2} u_{-}\right) \geq \operatorname{det}\left(D^{2} u_{R}\right) \quad \text { on } B_{R} \backslash \bar{B}_{1}
$$


and

$$
\operatorname{det}\left(D^{2} u_{-}\right)>\operatorname{det}\left(D^{2} u_{R}\right) \quad \text { on } B_{1},
$$

we have, by the maximum principle, $|\bar{x}|=1$. But this is impossible in view of (4.3) and the smoothness of $u_{R}$. The first inequality in (4.7) is established.

By Lemma 4.1, we can apply Pogorelov estimates and then Evans-Krylov estimates and Schauder theory to $u_{R}$ to obtain that on every compact subset $K$ of $\mathbb{R}^{n}$,

$$
\left\|u_{R}\right\|_{C^{k}(K)} \leq C(K, k) \quad \forall k
$$

So along a subsequence $R_{i} \rightarrow \infty$,

$$
u_{R_{i}} \rightarrow u \quad \text { in } C_{\mathrm{loc}}^{k}\left(\mathbb{R}^{n}\right) \quad \forall k
$$

It follows that $u$ is convex and satisfies (1.3) and

$$
u_{-}(x)+\beta_{-} \leq u(x) \leq u_{+}(x)+\beta_{+} \text {on } \mathbb{R}^{n} .
$$

In particular, $u \in C^{\infty}\left(\mathbb{R}^{n}\right)$ and satisfies (1.3) and (4.2).

Without the additional smoothness hypothesis on $f$, let $f_{\epsilon}=f * \rho_{\epsilon}$, where $\rho_{\epsilon}$ is the usual mollifier. Let $u_{\epsilon}$ be the solution found above for $f_{\epsilon}$. We know from the proof that $\left.\left|u_{\epsilon}(x)-\frac{1}{2}\right| x\right|^{2} \mid \leq C$ on $\mathbb{R}^{n}$ for some $C$ depending only on $n$ and $f$. Then, by the convexity of $u_{\epsilon}$ and the above estimates, $\left\{\left|u_{\epsilon}\right|+\left|D u_{\epsilon}\right|\right\}$ is uniformly bounded on any compact subset of $\mathbb{R}^{n}$. Passing to a subsequence (still denoted as $\left.\left\{u_{\epsilon}\right\}\right), u_{\epsilon} \rightarrow u$ in $C_{\text {loc }}^{0}\left(\mathbb{R}^{n}\right)$ for some convex function $u$. So $u$ is a viscosity solution of (1.3) and satisfies (4.2).

By Theorem 1.2, there exist $b \in \mathbb{R}^{n}, c \in \mathbb{R}$, and $A \in \mathcal{A}$ such that (1.6) holds. In view of (4.2), $A=I$ and $b=0$. Replacing $u$ by $u-c$ (but still calling it $u$ ), clearly $E(x):=u(x)-\left[|x|^{2} / 2+c\right]$ satisfies (1.11). To see (1.12), we first deduce from (4.8) that

$$
|E(x)| \leq C, \quad x \in \mathbb{R}^{n} .
$$

Here and in the following, $C$ and $r$ denote various large positive constants depending only on $n$ and $f$. Applying Lemma 3.5, with $\gamma=0$, we have

$$
\left|D^{2} E(x)\right| \leq \frac{C}{|x|^{2}}, \quad|x| \geq r .
$$

Since $E$ satisfies

$$
\tilde{a}_{i j}(x) D_{i j} E=0 \text { on } \mathbb{R}^{n} \backslash \bar{B}_{1} \quad \text { with } \quad \tilde{a}_{i j}(x)=\int_{0}^{1} F_{\xi_{i j}}\left(I+s D^{2} E(x)\right) d s .
$$

In view of the above estimates on $D^{2} E$, the Green's function for $\left(\tilde{a}_{i j}(x)\right)$ is bounded by $C|x|^{2-n}$ for $|x| \geq r$. Estimate (1.12) then follows from (1.11) and the maximum principle. Estimate (1.13) follows from (1.12) and Lemma 3.5 with $\gamma=n-2$. Theorem 1.7 is established. 


\section{Proof of Theorem 1.5}

By an affine transformation and by subtracting a linear function from $u$, we only need to prove the theorem for $A=I, b=0$, and $B_{2}(0) \subset D$. These will be assumed below.

Lemma 5.1 There exists some constant $C$, depending only on $n, \varphi$, and $D$, such that, for every $\xi \in \partial D$, there exists $\bar{x}(\xi) \in \mathbb{R}^{n}$ satisfying

$$
|\bar{x}(\xi)| \leq C \quad \text { and } \quad w_{\xi}<\varphi \text { on } \partial D \backslash\{\xi\},
$$

where

$$
w_{\xi}(x):=\varphi(\xi)+\frac{1}{2}\left(|x-\bar{x}(\xi)|^{2}-|\xi-\bar{x}(\xi)|^{2}\right), \quad x \in \mathbb{R}^{n} .
$$

PROOF: Let $\xi \in \partial D$. By a translation and a rotation, we may assume without loss of generality that $\xi=0$ and $\partial D$ is locally represented by the graph of

$$
x_{n}=\rho\left(x^{\prime}\right):=\frac{1}{2} \sum_{1 \leq \alpha, \beta \leq n-1} B_{\alpha \beta} x_{\alpha} x_{\beta}+\circ\left(\left|x^{\prime}\right|^{2}\right),
$$

and $\varphi$ locally has the expansion

$$
\varphi\left(x^{\prime}, \rho\left(x^{\prime}\right)\right)=\varphi(0)+\varphi_{x_{1}}(0) x_{1}+\frac{1}{2} \sum_{1 \leq \alpha, \beta \leq n-1} A_{\alpha \beta} x_{\alpha} x_{\beta}+\circ\left(\left|x^{\prime}\right|^{2}\right),
$$

where $x^{\prime}=\left(x_{1}, \ldots, x_{n-1}\right)$ and $\left(B_{\alpha \beta}\right)$ is positive definite.

Let

$$
\bar{x}=\left(-\varphi_{x_{1}}(0), 0, \ldots, 0, \bar{x}_{n}\right)
$$

and

Then

$$
w(x)=\varphi(0)+\frac{1}{2}\left(|x-\bar{x}|^{2}-|\bar{x}|^{2}\right), \quad x \in \mathbb{R}^{n} .
$$

$$
w\left(x^{\prime}, \rho\left(x^{\prime}\right)\right)=\varphi(0)+\varphi_{x_{1}}(0) x_{1}+\frac{1}{2}\left[\left|x^{\prime}\right|^{2}+\rho\left(x^{\prime}\right)^{2}\right]-\bar{x}_{n} \rho\left(x^{\prime}\right) .
$$

It follows that

$$
\begin{aligned}
& (w-\varphi)\left(x^{\prime}, \rho\left(x^{\prime}\right)\right)= \\
& \quad \frac{1}{2}\left[\left|x^{\prime}\right|^{2}+\rho\left(x^{\prime}\right)^{2}\right]-\frac{1}{2} \sum_{1 \leq \alpha, \beta \leq n-1} A_{\alpha \beta} x_{\alpha} x_{\beta}-\bar{x}_{n} \rho\left(x^{\prime}\right)+\circ\left(\left|x^{\prime}\right|^{2}\right) .
\end{aligned}
$$

By the strict convexity of $\partial D$, there exists some constant $\delta>0$ depending only on $D$ such that

$$
\rho\left(x^{\prime}\right) \geq \delta\left|x^{\prime}\right|^{2} \quad \forall\left|x^{\prime}\right|<\delta .
$$

Clearly, for large $\bar{x}_{n}$, we have

$$
(w-\varphi)\left(x^{\prime}, \rho\left(x^{\prime}\right)\right)<0 \quad \forall 0<\left|x^{\prime}\right|<\delta .
$$


The largeness of $\bar{x}_{n}$ depends only on $\delta$ and $\varphi$.

On the other hand,

$$
w(x)=\varphi(0)+\frac{1}{2}|x|^{2}+\varphi_{x_{1}}(0) x_{1}-x_{n} \bar{x}_{n} .
$$

By the strict convexity of $\partial D$ and (5.1),

$$
x_{n} \geq \delta^{3} \quad \forall x \in \partial D \backslash\left\{\left(x^{\prime}, \rho\left(x^{\prime}\right)\right):\left|x^{\prime}\right|<\delta\right\} .
$$

It follows that

$$
w(x) \leq \varphi(0)+\frac{1}{2}|x|^{2}+\varphi_{x_{1}}(0) x_{1}-\delta^{3} \bar{x}_{n} \quad \forall x \in \partial D \backslash\left\{\left(x^{\prime}, \rho\left(x^{\prime}\right)\right):\left|x^{\prime}\right|<\delta\right\} .
$$

By making $\bar{x}_{n}$ larger (still under control), we have

$$
w(x)-\varphi(x)<0 \quad \forall x \in \partial D \backslash\left\{\left(x^{\prime}, \rho\left(x^{\prime}\right)\right):\left|x^{\prime}\right|<\delta\right\} .
$$

Lemma 5.1 is established.

Fix some $c_{1} \in \mathbb{R}^{n}$ such that

$$
w_{\xi}(x) \leq \frac{1}{2}|x|^{2}+c_{1} \quad \forall \xi \in \partial D, x \in \mathbb{R}^{n} \backslash D, \operatorname{dist}(x, \partial D) \leq 1 .
$$

For $x \in \mathbb{R}^{n} \backslash \bar{D}$, let $\mathcal{S}_{c, x}$ denote the set of functions in $C^{0}\left(\mathbb{R}^{n} \backslash D\right)$ that are locally convex viscosity subsolutions of

$$
\operatorname{det}\left(D^{2} w\right) \geq 1 \quad \text { in } \mathbb{R}^{n} \backslash \bar{D}
$$

satisfying

$$
w \leq \varphi \quad \text { on } \partial D
$$

and

$$
w(y) \leq \frac{1}{2}|y|^{2}+c \quad \forall y \in \mathbb{R}^{n} \backslash D,|y-x| \leq 2 \operatorname{diam}(D) .
$$

Clearly $\mathcal{S}_{c, x}$ is nonempty for all $x$ and $c$.

Define

$$
u_{c}(x)=\sup \left\{w(x): w \in \mathcal{S}_{c, x}\right\}, \quad x \in \mathbb{R}^{n} \backslash \bar{D} .
$$

LEMMA 5.2 We have

(i) $u_{c}(x) \leq \frac{1}{2}|x|^{2}+c, x \in \mathbb{R}^{n} \backslash \bar{D}$;

(ii) $u_{c}$ is a locally convex viscosity subsolution of $\operatorname{det}\left(D^{2} u_{c}\right)=1$ in $\mathbb{R}^{n} \backslash \bar{D}$;

(iii) $u_{c}$ can be extended to a continuous function on $\mathbb{R}^{n} \backslash D$ with $u_{c}=\varphi$ on $\partial D$; and

(iv) $u_{c}$ is a viscosity solution of $\operatorname{det}\left(D^{2} u_{c}\right)=1$ on $\mathbb{R}^{n} \backslash \bar{D}$.

PROOF: (i) follows from the definition since $w(x) \leq \frac{1}{2}|x|^{2}+c$ for all $w \in \mathcal{S}_{c, x}$. (ii) holds since $u_{c}$ locally is the sup over a family of convex viscosity subsolutions.

For $\bar{\xi} \in \partial D$ and $x$ close to $\bar{\xi}$, since $w_{\xi} \in \mathcal{S}_{c, x}$ for all $\xi \in \partial D$, we have $u_{c}(x) \geq w_{\xi}(x)$ for $x$ close to $\bar{\xi}$. It follows that $\liminf _{x \rightarrow \bar{\xi}} u_{c}(x) \geq \varphi(\bar{\xi})$. On the other hand, $\lim \sup _{x \rightarrow \bar{\xi}} u_{c}(x) \leq \varphi(\bar{\xi})$. Indeed, if along a sequence $x_{i} \rightarrow \bar{\xi}$, 
$\lim _{i \rightarrow \infty} u_{c}\left(x_{i}\right) \geq \varphi(\bar{\xi})+3 \delta$ for some $\delta>0$. Then by the definition of $u_{c}$, there exists $w_{i} \in \mathcal{S}_{c, x_{i}}$ such that $w_{i}\left(x_{i}\right) \geq \varphi(\bar{\xi})+2 \delta$ for large $i$. But $w_{i}$ is locally convex and, for $\xi$ close to $\bar{\xi}, w_{i}(\xi) \leq \varphi(\xi)<\varphi(\bar{\xi})+\delta$. This forces $w_{i}$ to be unbounded near $\bar{\xi}$, contradicting the fact that $w_{i} \in \mathcal{S}_{c, x_{i}}$. (iii) is established.

For $\bar{x} \in \mathbb{R}^{n} \backslash \bar{D}$, fix some $\epsilon$ satisfying $0<\epsilon<2 \operatorname{diam}(D)$ and $B_{\epsilon}(\bar{x}) \subset \mathbb{R}^{n} \backslash \bar{D}$. By the definition of $u_{c}$,

$$
u_{c}(y) \leq \frac{1}{2}|y|^{2}+c \quad \forall|y-\bar{x}| \leq \epsilon .
$$

It is well-known (see, e.g., the appendix) that there is a unique convex viscosity solution $\tilde{u} \in C^{0}\left(\overline{B_{\epsilon}(\bar{x})}\right)$ to

$$
\begin{cases}\operatorname{det}\left(D^{2} \tilde{u}\right)=1 & \text { in } B_{\epsilon}(\bar{x}) \\ \tilde{u}=u_{c} & \text { on } \partial B_{\epsilon}(\bar{x}) .\end{cases}
$$

By the maximum principle, $\tilde{u} \geq u_{c}$ on $B_{\epsilon}(\bar{x})$. Define

$$
w(y)= \begin{cases}\tilde{u}(y) & \text { if } y \in B_{\epsilon}(\bar{x}) \\ u_{c}(y) & \text { if } y \in \mathbb{R}^{n} \backslash\left(D \cup B_{\epsilon}(\bar{x})\right) .\end{cases}
$$

Clearly, $w \in \mathcal{S}_{c, x}$. So, by the definition of $u_{c}, u_{c} \geq w$ on $B_{\epsilon}(\bar{x})$. It follows that $u_{c} \equiv \tilde{u}$ on $B_{\epsilon}(\bar{x})$. (iv) is established.

For $b \in \mathbb{R}$ and $a>-1$, let

$$
w_{a, b}(x)=b+\int_{1}^{|x|}\left(s^{n}+a\right)^{1 / n} d s .
$$

Then $w_{a, b}$ is a locally convex smooth solution of

$$
\begin{cases}\operatorname{det}\left(D^{2} w_{a, b}\right)=1, & \mathbb{R}^{n} \backslash B_{1}, \\ w_{a, b}=b, & \partial B_{1} .\end{cases}
$$

Let $\bar{r}=2 \operatorname{diam}(D)$ and $b(a):=\min _{\partial D} \varphi-\int_{1}^{\bar{r}}\left(s^{n}+a\right)^{1 / n} d s ;$ clearly $w_{a, b(a)} \leq \varphi$ on $\partial D$. It is easy to see that

$$
\begin{aligned}
& \lim _{|x| \rightarrow \infty}\left(w_{a, b(a)}(x)-\frac{|x|^{2}}{2}\right) \\
& \quad=b(a)-\frac{1}{2}+\int_{1}^{\infty} s\left(\left(1+\frac{a}{s^{n}}\right)^{1 / n}-1\right) d s \\
& =\mu(a):=\min _{\partial D} \varphi-\frac{1}{2}-\int_{1}^{\bar{r}}\left(s^{n}+a\right)^{1 / n} d s+\int_{1}^{\infty} s\left(\left(1+\frac{a}{s^{n}}\right)^{1 / n}-1\right) d s r \\
& \quad=\min _{\partial D} \varphi-\frac{1}{2}-\frac{1}{2}\left(\bar{r}^{2}-1\right)+\int_{\bar{r}}^{\infty} s\left(\left(1+\frac{a}{s^{n}}\right)^{1 / n}-1\right) d s .
\end{aligned}
$$

Clearly $\mu(a)$ is smooth, strictly monotonically increasing, and $\mu(a) \rightarrow \infty$ as $a \rightarrow \infty$. 
Fix some $a_{*}>-1$ such that $c_{*}:=\mu\left(a_{*}\right) \geq c_{1}$.

LEMMA 5.3 For $c>c_{*}$,

$$
\liminf _{|x| \rightarrow \infty}\left(u_{c}(x)-\frac{1}{2}|x|^{2}\right) \geq c
$$

PROOF: For all $\mu^{-1}\left(c_{*}\right)<a<\mu^{-1}(c)$,

$$
\lim _{|x| \rightarrow \infty}\left(w_{a, b(a)}(x)-\frac{|x|^{2}}{2}\right)=\mu(a)<c .
$$

It follows that $w_{a, b(a)} \in \mathcal{S}_{c, x}$ for sufficiently large $|x|$. It follows that $u_{c}(x) \geq$ $w_{a, b(a)}(x)$ and therefore

$$
\liminf _{|x| \rightarrow \infty}\left(u_{c}(x)-\frac{|x|^{2}}{2}\right) \geq \mu(a) .
$$

Lemma 5.3 follows after sending $a$ to $\mu^{-1}(c)$.

Proof of TheOREM 1.5: It follows from Lemma 5.2, Lemma 5.3, and Theorem 1.2.

\section{Appendix}

The following lemma and its proof can be found in [3]. For the reader's convenience, we include them here.

LEMMA A.1 Let $\Omega$ be a convex open set with $\operatorname{diam}(\Omega) \leq 1$, and let $u \in C^{0}(\bar{\Omega})$ be a convex viscosity solution of

$$
\begin{cases}\operatorname{det}\left(D^{2} u\right) \leq 1 & \text { in } \Omega \\ u \geq 0 & \text { on } \partial \Omega\end{cases}
$$

Then

$$
u(x) \geq \begin{cases}-C(n) \operatorname{dist}(x, \partial \Omega)^{2 / n} & \forall x \in \Omega, n \geq 3 \\ -C(\alpha) \operatorname{dist}(x, \partial \Omega)^{\alpha} & \forall x \in \Omega, n=2,0<\alpha<1\end{cases}
$$

PROOF: Pick a point on $\partial \Omega$, call it the origin 0 , and then let the $x_{n}$-axis point in the inward normal direction of $\partial \Omega$. Let

$$
h(x)= \begin{cases}\left(\left|x^{\prime}\right|^{2}-C\right) x_{n}^{2 / n}, & n \geq 3 \\ \left(x_{1}^{2}-C\right) x_{2}^{\alpha}, & n=2\end{cases}
$$

where $0<\alpha<1$. As in [3], for $C$ suitably large (depending only on $n$ when $n \geq 3$, while depending only on $\alpha$ when $n=2$ ), $h$ satisfies

$$
\left(D^{2} h\right)>0, \quad \operatorname{det}\left(D^{2} h\right) \geq 1 \text { on } \Omega, \quad \text { and } h \leq 0 \text { on } \partial \Omega .
$$


By the maximum principle,

$$
h \leq u \quad \text { on } \Omega
$$

in particular,

$$
u\left(x^{\prime}, x_{n}\right) \geq h\left(x^{\prime}, 0\right), \quad\left(x^{\prime}, x_{n}\right) \in \Omega .
$$

Lemma A.1 follows.

LEMMA A.2 Let $B$ be a ball in $\mathbb{R}^{n}, n \geq 2$, and let $f$ be a positive continuous function on $\bar{B}$. Assume that $u$ and $v$ are convex continuous functions on $\bar{B}$ that satisfy, in the viscosity sense,

$$
\operatorname{det}\left(D^{2} u\right) \leq f \text { in } B \text { and } \operatorname{det}\left(D^{2} u\right) \geq f \text { in } B .
$$

Assume also that

$$
u \geq v \quad \text { on } \partial B
$$

Then

$$
u \geq v \text { on } \bar{B}
$$

PROOF: We may assume without loss of generality that $u>v$ on $\partial B$. Indeed, we may consider $u+\epsilon$ for $\epsilon>0$ and then let $\epsilon$ tend to zero. We prove it by contradiction. Suppose the contrary; for some $\bar{x} \in B$,

$$
(u-v)(\bar{x})=\min _{\bar{B}}(u-v)<0 .
$$

Let $\left\{f_{j}^{-}\right\},\left\{f_{j}^{+}\right\} \subset C^{\infty}(\bar{B})$, satisfy

$$
f_{j}^{-}>f>f_{j}^{+} \text {on } \bar{B} \text { and } f_{j}^{ \pm} \rightarrow f \text { in } C^{0}(\bar{B}) .
$$

Let $\varphi \in C^{\infty}(\partial B)$ satisfy $v+\epsilon<\varphi<u-\epsilon$ on $\partial B$ where $3 \epsilon=\min _{\partial B}(u-v)>0$. By Proposition 2.4 , let $w_{j}^{ \pm} \in C^{0}(\bar{B}) \cap C^{\infty}(B)$ be strictly convex solutions of

$$
\begin{cases}\operatorname{det}\left(D^{2} w_{j}^{ \pm}\right)=f_{j}^{ \pm} & \text {in } B \\ w_{j}^{ \pm}=\varphi & \text { on } \partial B .\end{cases}
$$

Using $w_{j}^{ \pm}$as test functions in the definition of viscosity solutions, we have

$$
u-\epsilon \geq w_{j}^{-} \text {on } \bar{B} \quad \text { and } \quad w_{j}^{+} \geq v+\epsilon \text { on } \bar{B} \text {; }
$$

in particular,

$$
u(\bar{x})-\epsilon \geq w_{j}^{-}(\bar{x}), \quad w_{j}^{+}(\bar{x}) \geq v(\bar{x})+\epsilon .
$$

Since $u(\bar{x})<v(\bar{x})$, we have

$$
w_{j}^{+}(\bar{x}) \geq w_{j}^{-}(\bar{x})+2 \epsilon .
$$

By the Alexandrov estimate and (A.1), we have

$$
2 \epsilon \leq-\min _{\bar{B}}\left(w_{j}^{-}-w_{j}^{+}\right) \leq C\left(\int_{S_{j}^{+}} \operatorname{det}\left(D^{2}\left(w_{j}^{-}-w_{j}^{+}\right)\right)\right)^{1 / n},
$$


where $S_{j}^{+}=\left\{x \in B: D^{2}\left(w_{j}^{-}-w_{j}^{+}\right)(x)>0\right\}$.

Write

$$
\frac{D^{2} w_{j}^{-}(x)}{2}=\frac{\left[D^{2} w_{j}^{-}(x)-D^{2} w_{j}^{+}(x)\right]+\left[D^{2} w_{j}^{+}(x)\right]}{2} .
$$

For $x \in S_{j}^{+}, D^{2} w_{j}^{-}(x)-D^{2} w_{j}^{+}(x)$ is positive definite, so by the concavity of $(\mathrm{det})^{1 / n}$, we have

$$
\begin{aligned}
\left(\operatorname{det}\left[\frac{D^{2} w_{j}^{-}(x)}{2}\right]\right)^{1 / n} & \geq \\
& \frac{1}{2}\left(\operatorname{det}\left[D^{2} w_{j}^{-}(x)-D^{2} w_{j}^{+}(x)\right]\right)^{1 / n}+\frac{1}{2}\left(\operatorname{det}\left[D^{2} w_{j}^{+}(x)\right]\right)^{1 / n},
\end{aligned}
$$

i.e.,

$$
\frac{1}{2} f_{j}^{-}(x)^{1 / n} \geq \frac{1}{2}\left(\operatorname{det}\left[D^{2} w_{j}^{-}(x)-D^{2} w_{j}^{+}(x)\right]\right)^{1 / n}+\frac{1}{2} f_{j}^{+}(x)^{1 / n} .
$$

Since $f_{j}^{ \pm} \rightarrow f$ in $C^{0}(\bar{B})$, we have

$$
\sup _{x \in S_{j}^{+}} \operatorname{det}\left[D^{2} w_{j}^{-}(x)-D^{2} w_{j}^{+}(x)\right] \rightarrow 0 .
$$

Sending $j$ to $\infty$ in (A.2), we have $2 \epsilon \leq 0$, which is a contradiction.

LemmA A.3 Let $B$ be a ball of $\mathbb{R}^{n}$ and $\varphi \in C^{0}(\partial B)$. Assume that $\underline{u} \in C^{0}(\bar{B})$ is a convex viscosity subsolution to $\operatorname{det}\left(D^{2} \underline{u}\right) \geq 1$. Then

$$
\begin{cases}\operatorname{det}\left(D^{2} u\right)=1 & \text { in } B \\ u=\underline{u} & \text { on } \partial B\end{cases}
$$

has a unique convex viscosity solution $u \in C^{0}(\bar{B})$.

Remark A.4. The same conclusion holds when replacing $B$ by any bounded convex open set of $\mathbb{R}^{n}$.

PROOF: Uniqueness follows from the maximum principle. Let $\varphi_{i} \in C^{\infty}(\partial B)$ satisfy

$$
\underline{u}<\varphi_{i} \leq \underline{u}+\frac{1}{i} \text { on } \partial B \text { and } \varphi_{i} \rightarrow \underline{u} \text { in } C^{0}(\partial B) .
$$

It follows from [6] that there exists a unique, strictly convex solution $u_{i} \in C^{\infty}(\bar{B})$ of $\operatorname{det}\left(D^{2} u_{i}\right)=1$ in $B$ and $u_{i}=\varphi_{i}$ on $\partial B$. By the maximum principle, $\underline{u} \leq u_{i} \leq h_{i}$ on $\bar{B}$, where $h_{i}$ is the harmonic function on $B$ with boundary value $\varphi_{i}$. By the convexity and the uniform bound of $\left\{u_{i}\right\},\left|\nabla u_{i}\right|$ is bounded on compact subsets of $B$. So, after passing to a subsequence, $u_{i}$ uniformly converges on compact subsets of $B$ to some convex function $u \in C^{0}(B)$. Consequently, $u$ is a viscosity solution to $\operatorname{det}\left(D^{2} u\right)=1$. Clearly, $\underline{u} \leq u \leq h$ on $B$, where $h$ is the harmonic function on $B$ with boundary value $h=\underline{u}$. It follows that $u$ can be extended as a continuous function on $\bar{B}$ with $u=\underline{u}$ on $\partial B$. Lemma A. 3 is established. 
The following is a linear algebra lemma.

LEMMA A.5 Let $U$ be an $n \times n$ real upper-triangular matrix. Assume that the diagonals of $U$ are nonnegative and, for some $0<\epsilon<1$,

$$
(1-\epsilon) B \subset U(B) \subset(1+\epsilon) B,
$$

where $B \subset \mathbb{R}^{n}$ is the unit ball centered at the origin. Then for some constant $C=C(n)$,

$$
\|U-I\| \leq C \epsilon
$$

ProOF: Letting $U=\left(U_{i j}\right)$, we know that $U_{i j}=0$ for $i<j$. Since $U(B)$ contains an open neighborhood of $\mathbb{R}^{n}, U$ is invertible, and therefore $U_{i i}>0 \forall i$. Write $U^{-1}=\left(U^{i j}\right)$; then $U^{-1}$ is also upper-triangular, $U^{i i}=1 / U_{i i}$ for every $i$, and, by (A.4),

$$
\frac{1}{1+\epsilon} B \subset U^{-1}(B) \subset \frac{1}{1-\epsilon} B .
$$

For $1 \leq k \leq n$, let $e_{k}$ denote the unit vector with the $k^{\text {th }}$ component equal to 1 and all the other components equal to zero. By (A.4)

$$
\left\|U e_{k}\right\|=\sqrt{\sum_{j=1}^{n} U_{j k}^{2}} \leq 1+\epsilon
$$

in particular, $U_{k k} \leq 1+\epsilon, 1 \leq k \leq n$. The same argument can be applied to $U^{-1}$, so, in particular,

We deduce from the above two estimates that

$$
\frac{1}{U_{k k}}=U^{k k} \leq \frac{1}{1-\epsilon}, \quad 1 \leq k \leq n .
$$

$$
1-\epsilon \leq U_{k k} \leq 1+\epsilon, \quad 1 \leq k \leq n .
$$

It follows from (A.6) and (A.7) that

$$
\sum_{j \neq k} U_{j k}^{2} \leq(1+\epsilon)^{2}-(1-\epsilon)^{2}=4 \epsilon, \quad 1 \leq k \leq n .
$$

Estimate (A.5) follows from (A.7) and (A.8).

\section{Bibliography}

[1] Aubin, T. Équations de Monge-Ampère réelles. J. Funct. Anal. 41 (1981), no. 3, 354-377.

[2] Caffarelli, L. A. Interior $W^{2, p}$ estimates for solutions of the Monge-Ampère equation. Ann. of Math. (2) 131 (1990), no. 1, 135-150.

[3] Caffarelli, L. A. A localization property of viscosity solutions to the Monge-Ampère equation and their strict convexity. Ann. of Math. (2) 131 (1990), no. 1, 129-134.

[4] Caffarelli, L. A. Topics in PDEs: The Monge-Ampère equation. Graduate course. Courant Institute, New York University, 1995. 
[5] Caffarelli, L. A.; Cabré, X. Fully nonlinear elliptic equations. American Mathematical Society Colloquium Publications, 43. American Mathematical Society, Providence, R.I., 1995.

[6] Caffarelli, L.; Nirenberg, L.; Spruck, J. The Dirichlet problem for nonlinear second-order elliptic equations. I. Monge-Ampère equation. Comm. Pure Appl. Math. 37 (1984), no. 3, 369-402.

[7] Calabi, E. Improper affine hyperspheres of convex type and a generalization of a theorem by K. Jörgens. Michigan Math. J. 5 (1958), 105-126.

[8] Cheng, S. Y.; Yau, S. T. On the regularity of the Monge-Ampère equation $\operatorname{det}\left(\partial^{2} u / \partial x_{i} \partial x_{j}\right)=$ F (x, u). Comm. Pure Appl. Math. 30 (1977), no. 1, 41-68.

[9] Cheng, S. Y.; Yau, S. T. Complete affine hypersurfaces. I. The completeness of affine metrics. Comm. Pure Appl. Math. 39 (1986), no. 6, 839-866.

[10] Chou, K.-S.; Wang, X.-J. Entire solutions of the Monge-Ampère equation. Comm. Pure Appl. Math. 49 (1996), no. 5, 529-539.

[11] de Guzmán, M. Differentiation of integrals in $R^{n}$. Lecture Notes in Mathematics, 481. Springer, Berlin-New York, 1975.

[12] Delanoë, P. Partial decay on simple manifolds. Ann. Global Anal. Geom. 10 (1992), no. 1, 3-61.

[13] Ferrer, L.; Martínez, A.; Milán, F. An extension of a theorem by K. Jörgens and a maximum principle at infinity for parabolic affine spheres. Math. Z. 230 (1999), no. 3, 471-486.

[14] Ferrer, L.; Martínez, A.; Milán, F. The space of parabolic affine spheres with fixed compact boundary. Monatsh. Math. 130 (2000), no. 1, 19-27.

[15] Gilbarg, D.; Serrin, J. On isolated singularities of solutions of second order elliptic differential equations. J. Analyse Math. 4 (1955/56), 309-340.

[16] Gilbarg, D.; Trudinger, N. S. Elliptic partial differential equations of second order. Second edition. Grundlehren der Mathematischen Wissenschaften, 224. Springer, Berlin, 1983.

[17] Jörgens, K. Über die Lösungen der Differentialgleichung $r t-s^{2}=1$. Math. Ann. 127 (1954), 130-134.

[18] Pogorelov, A. V. On the improper convex affine hyperspheres. Geometriae Dedicata 1 (1972), no. $1,33-46$.

[19] Trudinger, N. S.; Wang, X.-J. The Bernstein problem for affine maximal hypersurfaces. Invent. Math. 140 (2000), no. 2, 399-422.

\section{CAFFARELLI}

University of Texas

Department of Mathematics

Austin, TX 78712

E-mail: caffarele math.utexas. edu

\section{YANYAN LI}

Rutgers-The State University

Department of Mathematics

New Brunswick, NJ 08903

E-mail: yyli@math.rutgers.edu

\section{Received November 2001.}

